\title{
A moment model for phonon transport at room temperature
}

\section{Alireza Mohammadzadeh* and Henning Struchtrup}

(Dated: August 4, 2016)

Heat transfer in solids is modeled by deriving the macroscopic equations for phonon transport from the phonon-Boltzmann equation. In these equations, the Callaway model with frequency dependent relaxation time is considered to describe the Resistive and Normal processes in the phonon interactions. Also, the Brillouin zone is considered to be a sphere, its diameter depends on the temperature of the system. A simple model to describe phonon interaction with crystal boundary is employed to obtain macroscopic boundary conditions, where the reflection kernel is the superposition of diffusive reflection, specular reflection and isotropic scattering. Macroscopic moments are defined using a polynomial of the frequency and wave vector of phonons. As an example, a system of moment equations, consisting of 3 directional and 7 frequency moments, i.e., 63 moments in total, is used to study one-dimensional heat transfer, as well as Poiseuille flow of phonons. Our results show the importance of frequency dependency in relaxation times and macroscopic moments to predict rarefaction effects. Good agreement with data reported in the literature is obtained. 


\section{INTRODUCTION}

Miniaturization of devices in the last 50 years has attracted the attention of many researchers [1]. The rapid development of micro/nano electro mechanical systems (MEMS/NEMS) requires a deeper understanding of the flow characteristics and heat transfer mechanisms at micro/nano scales. Effects that are negligible at macro scales can dominate at micro and nano scales. For example, the characteristic length of a flow can become small enough that surface effects at interfaces dominate over the bulk properties of the flow, and subsequently lead to a breakdown at the classical laws of continuum mechanics.

The Knudsen number [2] is defined as the ratio of the mean free path $(\lambda)$ to the characteristic length of flow $(L)$, $\mathrm{Kn}=\frac{\lambda}{L}$, and indicates the degrees of rarefaction. The classical assumptions in continuum mechanics [2] start to break down as Kn becomes larger than 0.001 .

One important area of research in nano-technology is the study of heat transport. The tiny size of modern electronic systems combined with the relative large power of lasers can lead to huge changes in temperature. Therefore, we need accurate models for heat transfer that guarantee an effective long-lasting thermal design of modern micro/nano scale systems [3].

Heat transport in solids is due to the exchange of energy between the particles that vibrate on the crystal lattice, with respect to their mean position $[4,5]$. These vibrations lead to an energy wave inside the solid, that can be quantized into particles, known as phonons. The macroscopic properties of a crystal, such as temperature, internal energy and heat flux can be obtained by taking suitable averages of phonons properties, such as energy and momentum $[4]$.

Phonons can interact among themselves, and with other particles, including photons, electrons and crystal boundaries. In phonon-phonon collisions, phonons always conserve energy, but can lose momentum [4]. The interactions in which phonons conserve both energy and momentum are called Normal processes. The phonon processes in which the momentum does not remain conserved are called Resistive processes [4, 5].

Based on the magnitude of the Knudsen numbers for Resistive and Normal processes, we distinguish three modes of heat transfer in solids:

- The diffusive heat transfer, that dominates the heat transfer mechanisms when the mean free path for Resistive processes is much smaller than the characteristic length of the flow, $\lambda_{R} \ll L$. In this mode, phonons mainly undergo interactions that do not conserve momentum, as a results, the energy waves are damped in a very short length. In this regime the Fourier law governs the heat transfer process [4].

- The second mechanism happens when the mean free path for the Resistive process is much larger than the characteristic length of the flow $\lambda_{R} \gg L$, while the mean free path for the Normal process is smaller or comparable with the characteristic length of the flow, $\lambda_{N} \lesssim L$. In this regime, Normal processes by conserving the phonon momentum dominates the heat transfer mechanism, and leads to a wave like energy transport known as second sound $[6,7]$.

- The energy can also be transported in solids via ballistic phonons [8]. In this regime the mean free path for Resistive and Normal process are both larger than the characteristic length of the flow, i.e., $\lambda_{R}, \lambda_{N}>L$. In this Knudsen flow, phonons travel inside the crystal without any interactions.

In general, the energy transport in solids is a combination of the above mechanisms. Depending on the temperature range and the crystal properties, each of the above transport regimes can dominate.

The phonon-Boltzmann equation, first formulated by Peierls [9], describes the transport of phonons in the crystal lattice. This equation relates the time evolution of the phonon distribution function to phonon convection and collisions with other phonons, as well as other particles. While the phonon-Boltzmann equation is valid for all degrees of rarefaction, its direct solution, due to the complexity in the phase-space dimension as well as the non-linearity in the collision term, is a formidable task [10].

As an alternative to the direct solution, one can use discrete particle methods to track each individual phonon in the crystal, and use the deterministic approach (a branch of molecular dynamics) to study the phonon transport [11]. Another approach, known as direct simulation Monte Carlo (DSMC), is using statistical assumptions to group a cloud of phonons, and study their behavior [2]. Employing the stochastic assumptions in the DSMC method compared to the deterministic nature of molecular dynamics significantly reduces the computational costs. However, DSMC still suffers from expensive computational overheads [12]. More recently, a variance-reduction formulation has been proposed to improve the traditional Monte Carlo method, when the temperature difference is small [13]. In this method, the stochastic particle description is only solved for the deviation from a nearby equilibrium, that leads to significant speed up compared to standard DSMC. Peraud and Hadjiconstantinou [14] also showed that in the case 
where the governing deviational formulation for solving phonon-Boltzmann equation can be linearized, additional speed up will be obtained, which provides a suitable algorithm for using DSMC in three-dimensional geometries.

Due to the complexity of the collision term in the phonon-Boltzmann equation, the well-known Callaway model [15] was proposed to provide an approximation for this term. In this model, the collision is described as a process that relaxes the distribution function of phonons to the appropriate equilibrium state for collision type, with the relaxation time $\tau$ that needs to be provided.

When the mean free path for the Resistive process is very smaller than the length scale of the flow, i.e., $\mathrm{Kn}_{R} \ll 1$, by performing the Chapman-Enskog expansion [16] on the phonon-Boltzmann equation equipped with the Callaway model, one can obtain the Fourier law as the heat flux equation. The Fourier law provides an explicit expression for the heat conductivity, which can be determined by experimental measurements. However, since the second sound and ballistic phonons are not included in the Fourier law, it cannot provide satisfactory results when diffusion does not dominate the heat transport process [17-20].

By considering certain ratios between Resistive and Normal relaxation times, researchers derived macroscopic equations to extend the validity of classical hydrodynamics beyond the Fourier regime. Guyer and Krumhansl [8, $21,22]$ solved the linearized form of the phonon-Boltzmann equation in terms of eigenvectors of the Normal process when the ratio of Resistive and Normal processes are very small or very large. They showed that this condition leads to the Fourier law in the former, while a set of equations that can capture the second sound was obtained in the latter. Although these equations predicted the second sound effect, they were only valid at low temperature where the employed assumptions held.

Another approach to obtain a system of macroscopic equations with extended validity beyond the Fourier's regime is using higher order moments of the distribution function. This method, known as moment method, has been successful in rarefied gas dynamics, and shown to be capable of predicting and explaining the flow behavior up to mid-transition regime $(K n \lesssim 0.5)$. In the seminal work of Grad, a generic form of macroscopic moments was considered, and a set of transport equations were derived by taking integral moments of the Boltzmann equation [23, 24]. This approach provides an infinite number of transport equations for macroscopic moments, where in theory, its solution is equivalent to the solution of Boltzmann equation. However, in order to make this system of infinite number of equations practical, we need to truncate it at some point. Using Grad-type distribution function to provide closure for the system of moments is a common approach; however, this closure brings approximation to the system [23, 25]. In this method, the Knudsen number determines degrees of rarefaction in the flow, and subsequently, the required extra terms and equations to capture non-equilibrium. A big advantage of accessing to macroscopic equations compared to particle methods is the opportunity to relate non-equilibrium phenomena to macroscopic terms. These macroscopic terms in the system of moments can be simply switched on and off so that we study their effect to help us explain the non-equilibrium phenomena. So far, we had a great success in capturing and explaining thermal driven flows by system of moments in rarefied gas dynamics [26-28].

Struchtrup and co-workers [6, 29] followed Grad's moments method, and presented a simple model for the phonon transport in solids. Although this model described the interplay between Normal and Resistive processes, and included the second sound and ballistic phonon effects, due to its simplifying assumptions it could not provide good agreement with experimental data. The main simplifications considered in their model were (a) linear dispersion relation between phonon energy and momentum, (b) extension of the Brillouin zone to infinity, and (c) considering a constant relaxation time for the Callaway model. These simplifications provided fast access to a system of moments that could describe the rarefied phonon gas in principle, but fail to give accurate results at room temperature.

By aiming to access to a system of moments that can indeed capture the phonon transport at room temperature, we replace the simplifying assumptions employed in Ref. [29] with a more realistic model. We consider a quadratic dispersion relation to accurately describe the dependency of frequency on wavevector at room temperature. Moreover, the Brillouin zone is considered to be a sphere with finite radius, which depends on the working temperature of the system. Most importantly, the dependency of relaxation time on frequency is considered in the Callaway model, to account for phonon interactions at low and high frequencies.

Macroscopic moments are defined using a polynomial of frequency and wavevector of phonons. Using the aforementioned assumptions, the resulting transport equations for macroscopic moments are derived, and presented. The finite size of Brillouin zone as well as the frequency dependency in relaxation time makes the derivation of the transport equations more complicated compared to Ref. [29]. In particular, providing a closed form of integration at room temperature becomes a formidable task, and most integrations have to be numerically calculated.

In order to describe the phonon-surface interactions, we used an extension to the boundary condition model presented in Ref. [29]. In this model, three types of interactions are considered: thermalization, specular reflection and isotropic scattering. Based on the proportions of these three types, the free parameters in the reflection model can be altered to fit to the experimental data.

In order to fix the free parameters in our model, we use the experimental data for specific heat and thermal conductivity. We use the specific heat to fix the dispersion relation, and then by employing this relation, we use the 
thermal conductivity to fix the relaxation time in the production term. Then, the resulting equations are used to analytically solve the heat transfer problem in two simple geometries.

As the first application we model the thermal grating experiment [30], considering infinite width for the silicon specimen to neglect the effects of boundary conditions. This assumption leads to a one-dimensional flow problem, that can be solved using eigenvalue-eigenvector analysis. We compare the predicted results of our system with the reported data in Ref. [30]. The results show the importance of considering low frequency phonons as well as high frequency phonons in the relaxation time model, to get a good agreement with the reported data in Ref. [30]. Then, we use the system of moments to solve Poiseuille flow of phonons in a heat conductor with adiabatic boundaries. In this problem, the effect of boundary conditions on the heat transfer is studied. Moreover, we show the deviation of our results from the Fourier's solution, and discuss the role of relaxation time model on this deviation.

The remainder of the paper is organized as follows: In Sec. II we recall the kinetic theory of phonons, and introduce the dispersion relation as well as the specific heat. Then we do a quick review on the Callaway model, and introduce the relaxation times considered in this study. Macroscopic moments are presented in Sec. III, and the system of moment equations are derived from the phonon-Boltzmann equation. This system is then closed using the Grad distribution function in Sec. IIIB. The details of phonon-boundary interactions are presented in Sec. IV. Then, the closed system of moment equations as well as their boundary conditions, and the thermal conductivity predicted by this system is presented in Sec. V. In Sec. VI the system of moments are used in two simple geometries to solve heat transfer problems. The paper closes with conclusion is Sec. VII.

\section{PHONONS}

\section{A. Phonon model}

The energy is transported in solids when atoms vibrate around their mean positions on the crystal lattice. The particle representative of the resulting energy wave due to these vibrations is called phonon. Phonons have energy and momentum; they can interact among themselves and with other particles, including photons, electrons, and crystal boundaries. They can also interact with crystal impurities and dislocations and lose momentum [4]. The thermal properties of the crystal, such as temperature and heat flux can be obtained by taking appropriate averages of microscopic properties of the phonon gas.

In the phonon model the crystal is replaced by a box containing the gas of phonons, such that the energy transport can be treated analogous to the transport processes in gases [31,32]. The eigen-vibration of the lattice with the frequency $\omega$ and the wave vector $\mathbf{k}$ in the crystal corresponds to phonons with the energy $\hbar \omega$ and the momentum $\hbar \mathbf{k}$, where $\hbar$ is Planck's constant. The dispersion relation $\omega(\mathbf{k})$ determines the relationship between the frequency and the wave vector, which follows from the quantum mechanics equation of motion for atoms in the crystal [4]. This relation is periodic in nature, with a full period equal to the Brillouin zone. Phonons travel with the group velocity, $\mathbf{c}=\frac{\partial \omega}{\partial \mathbf{k}}$.

In contrast to the transport process for gas molecules, phonons do not follow the law of number conservation; they can be created or destroyed during interactions. The energy, on the other hand, is always conserved in the phonon-phonon interaction [9],

$$
\hbar \omega^{\prime}+\hbar \omega^{\prime \prime}=\hbar \omega^{\prime \prime \prime} \text { or } \hbar \omega^{\prime}=\hbar \omega^{\prime \prime}+\hbar \omega^{\prime \prime \prime} \text {. }
$$

In this process two phonons with frequencies $\omega^{\prime}$ and $\omega^{\prime \prime}$ interact, and create a phonon with the frequency $\omega^{\prime \prime \prime}$, or a phonon with frequency $\omega^{\prime}$ splits into two phonons with frequencies $\omega^{\prime \prime}$ and $\omega^{\prime \prime \prime}$. Phonon momentum is generally not conserved, but obeys

$$
\hbar \mathbf{k}^{\prime}+\hbar \mathbf{k}^{\prime \prime}=\hbar \mathbf{k}^{\prime \prime \prime}+\hbar \mathbf{G} \quad \text { or } \hbar \mathbf{k}^{\prime}=\hbar \mathbf{k}^{\prime \prime}+\hbar \mathbf{k}^{\prime \prime \prime}+\hbar \mathbf{G}
$$

where $\mathbf{G}$ is the reciprocal lattice vector. The phonon momentum is conserved when the reciprocal lattice vector is zero. This interaction is called Normal process. If the interaction between phonons leads to a momentum vector outside the Brillouin zone, the non-zero reciprocal vector $\mathbf{G}$ brings back the momentum vector to the Brillouin zone. This type of process is called Umklapp (flip over). The phonons can also interact with the lattice imperfections and boundaries, and lose momentum while conserving energy. All processes that do not conserve momentum are called Resistive processes $[4,5]$.

We distinguish two different types of phonons: optical phonons and acoustic phonons. Optical phonons appear in crystals with more than one atom in the smallest unit cell, and only have high frequencies. Therefore, they can be neglected in single element crystals, and at moderate temperatures range [4]. Acoustic phonons, on the other hand, can have very low to high frequencies. Acoustic phonons have 3 polarizations, 2 transversals and 1 longitudinal. In 
the current study we only consider acoustic phonons, and group their three polarizations into a single mode with a single velocity and single deformation potential [31].

\section{B. Kinetic theory of phonons}

In kinetic theory, the state of the phonon gas is described by the distribution function, $f(\mathbf{x}, \mathbf{k}, t)$, defined such that the number of phonons in a phase space element, $d \mathbf{k} d \mathbf{x}$ at time $t$ is given by

$$
d N=f(\mathbf{x}, \mathbf{k}, t) d \mathbf{k} d \mathbf{x} .
$$

Here, $\mathbf{x}$ is the three-dimensional space vector. The phonon-Boltzmann equation reads $[6,9]$

$$
\frac{\partial f}{\partial t}+c_{i} \frac{\partial f}{\partial x_{i}}=S_{R}(f)+S_{N}(f)
$$

where $c_{i}=\frac{\partial \omega}{\partial k_{i}}$ is the group velocity of phonons, and $S(f)$ is the collision operator including the Resistive $S_{R}(f)$ and the Normal process $S_{N}(f)$.

Moments of the distribution function provide the macroscopic properties of phonon system. The energy density, energy flux and momentum density in the phonon gas are obtained as

$$
\begin{aligned}
e(t, \mathbf{x}) & =\int_{B Z} \hbar \omega f(\mathbf{x}, \mathbf{k}, t) d \mathbf{k} \\
q_{i}(t, \mathbf{x}) & =\int_{B Z} \hbar \omega c_{i} f(\mathbf{x}, \mathbf{k}, t) d \mathbf{k} \\
p_{i}(t, \mathbf{x}) & =\int_{B Z} \hbar k_{i} f(\mathbf{x}, \mathbf{k}, t) d \mathbf{k}
\end{aligned}
$$

where $B Z$ denotes the Brillouin zone of the lattice. We assume that the dispersion relation only depends on the absolute value of the wave vector. This assumption leads to a spherical Brillouin zone [4] with the boundaries of

$$
-\frac{\pi}{a} \leq k_{i} \leq \frac{\pi}{a}
$$

where $a$ is the lattice spacing.

Phonons are Bose particles [4], and their entropy density reads

$$
s=-k_{B} \int_{B Z}\left(f \ln \left(\frac{f}{y}\right)-y\left(1+\frac{f}{y}\right) \ln \left(1+\frac{f}{y}\right)\right) d \mathbf{k},
$$

where $k_{B}$ is the Boltzmann's constant, and $y=\frac{3}{8 \pi^{3}}$ is the density of states [4]. Note that the factor 3 in this constant is related to the 3 phonon polarizations, with 2 branches for transversal and 1 branch for longitudinal modes. In the equilibrium state, phonons follow the Bose distribution function [4],

$$
f_{E}=\frac{y}{\exp \left(\frac{\hbar \omega}{k_{B} T}\right)-1} \text {. }
$$

\section{Dispersion relation and specific heat}

In order to relate the microscopic properties of phonons to the macroscopic properties of lattice, we need to express the dependency of frequency on the wave vector. We use an isotropic quadratic function, as suggested in Ref. [33], to approximate the dispersion relation as

$$
\omega(k)=v_{1} k+v_{2} k^{2}
$$

where $v_{1}$ and $v_{2}$ are constants. The group velocity is obtained by

$$
c_{i}=\frac{\partial \omega}{\partial k_{i}}=\left(v_{1}+2 v_{2} k\right) n_{i},
$$


where

$$
n_{i}=\frac{k_{i}}{k}
$$

is the phonon direction vector.

Specific heat is obtained by taking the temperature derivative of the energy moment in equilibrium, where $f$ is the Bose distribution function

$$
C=\frac{\partial e}{\partial T}=\frac{\partial\left(\int_{B Z} \hbar \omega f_{E} d \mathbf{k}\right)}{\partial T} .
$$

We set $v_{1}$ to the Debye speed $c_{0}$, and choose $v_{2}$ such that we get the best fit to the experimental values for specific heat at room temperature. The Debye speed $c_{0}$ is defined as

$$
\frac{1}{c_{0}^{3}}=\frac{1}{3} \sum_{i=1}^{3} \frac{1}{c_{i}^{3}}
$$

which is an appropriate average in the 3 polarizations for phonons [4]. Using literature [4] to set $c_{0}=5883 \frac{m}{s}$, we choose $v_{2}=-1.0103 \times 10^{-10} \frac{\mathrm{m}^{2}}{\mathrm{~s}}$ to fit to the experimental values for specific heat. Figure 1 shows the variation of specific heat with temperature obtained from the considered model, compared with the experimental data in Ref. [4]. The deviation that occurs around $T \simeq 100 K$ is due to the value of $v_{2}$ employed in the dispersion relation, that we chose to, specifically, fit to the values around room temperature. Note that we can extend the comparison to higher temperatures, however, in the current study we are interested in the room temperature comparison.

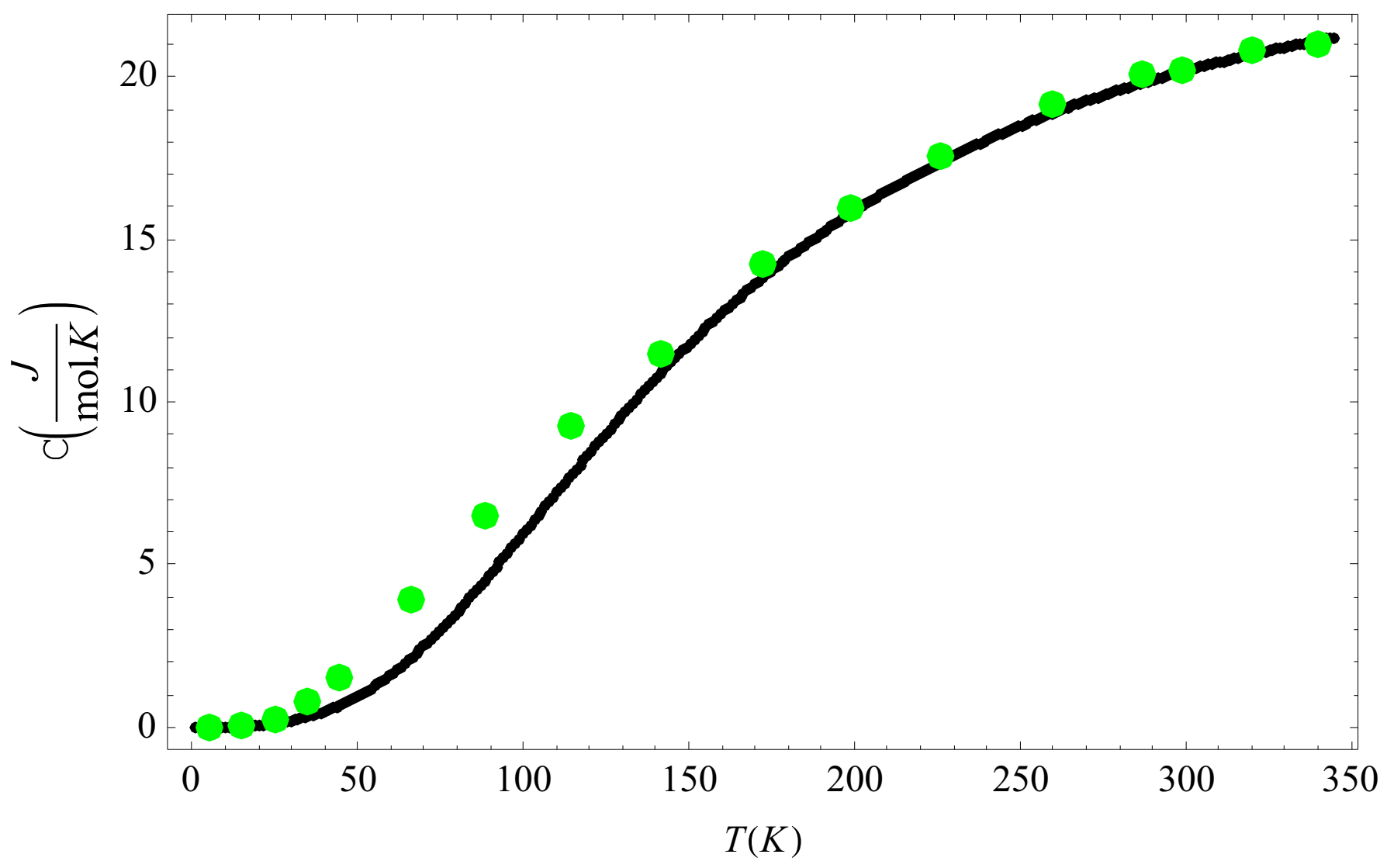

FIG. 1. Specific heat for Silicon predicted with the quadratic dispersion relation. 


\section{Callaway model}

We use the Callaway model [15] to describe the production term in the phonon-Boltzmann equation. This model is analogous to the BGK model in the kinetic theory of classical gases [34]. In this model, the phonon distribution function, $f$ relaxes to the reference distribution functions, $f_{R}$ and $f_{N}$, with the relaxation times $\tau_{R}$ and $\tau_{N}$ for the Resistive $(R)$ and Normal $(N)$ processes, respectively. The idea behind this model comes from the concept of maximum entropy at the equilibrium state. All phonon interactions are supposed to increase entropy, and drive the distribution function toward the equilibrium state corresponding to their type of interactions. The relaxation time relates to the phonon mean free path, and shows the rate of this increase in the flow. The Callaway model reads

$$
S(f)=-\frac{1}{\tau_{R}(\omega)}\left(f-f_{R}\right)-\frac{1}{\tau_{N}(\omega)}\left(f-f_{N}\right) .
$$

The equilibrium distributions $f_{R}$ and $f_{N}$, must be chosen to satisfy the conservation conditions for energy and momentum. In all $R$-processes the energy is conserved, hence

$$
\int_{B Z} \frac{\hbar \omega}{\tau_{R}(\omega)}\left(f-f_{R}\right) d \mathbf{k}=0
$$

and in all $N$-processes both energy and momentum are conserved,

$$
\int_{B Z} \frac{\hbar \omega}{\tau_{N}(\omega)}\left(f-f_{N}\right) d \mathbf{k}=0 \text { and } \int_{B Z} \frac{\hbar k_{i}}{\tau_{N}(\omega)}\left(f-f_{N}\right) d \mathbf{k}=0 .
$$

In order to obtain the appropriate expressions for the equilibrium distributions $f_{R}$ and $f_{N}$, we maximize the entropy density Eq. (4), under the constraints of Eq. (10) and Eq. (11), respectively. This leads to

$$
f_{R}=\frac{y}{e^{\frac{h \omega}{k_{B}} \gamma}-1} \text { and } f_{N}=\frac{y}{e^{\left(\frac{\hbar \omega}{k_{B}} \gamma^{\prime}+\frac{\xi_{j} h k_{j}}{k_{B}}\right)}-1},
$$

where $\gamma, \gamma^{\prime}$ and $\xi_{j}$ are the Lagrange multipliers. We assume that the phonon gas is not very far from the local equilibrium, so that the above distribution functions deviate only slightly from the local equilibrium distribution functions. This allows us to linearize these distribution function in Lagrange multipliers to get

$$
\begin{aligned}
& f_{R}=f_{E}+\left.\frac{\partial f_{R}}{\partial \gamma}\right|_{E}\left(\gamma-\gamma_{E}\right) \\
& f_{N}=f_{E}+\left.\frac{\partial f_{N}}{\partial \gamma^{\prime}}\right|_{E}\left(\gamma^{\prime}-\gamma_{E}^{\prime}\right)+\left.\frac{\partial f_{N}}{\partial \xi_{j}}\right|_{E}\left(\xi_{j}-\xi_{j, E}\right) .
\end{aligned}
$$

Note that by comparing Eq. (12) with the Bose distribution function, we have

$$
\gamma_{E}=\gamma_{E}^{\prime}=\frac{1}{T} \text { and } \xi_{j, E}=0
$$

The relaxation times in the Callaway model plays a very important role in predicting the phonon transport characteristics. The relaxation time for the $R$-processes reads [35]

$$
\frac{1}{\tau_{R}(\omega)}=\frac{1}{\tau_{U}(\omega)}+\frac{1}{\tau_{B}(\omega)}+\frac{1}{\tau_{X}(\omega)},
$$

where $\tau_{U}$ denotes the relaxation time for the Umklapp processes, $\tau_{B}$ denotes the relaxation time for the phononboundary interaction, and $\tau_{X}$ takes the effects of impurities into account. The effects of phonon-surface interactions will be considered in the boundary condition model in Sec. IV. Moreover, we consider a pure crystal without any imperfection, i.e., $\frac{1}{\tau_{X}(\omega)}=0$.

For the relaxation time in the Umklapp processes we use the results reported in Ref. [36]. Ward and Broido [36] employed the first principle approach to obtain the relaxation times, and observed that at low frequency $\tau_{U} \propto \frac{1}{\omega^{2}}$, while at high frequencies this dependency is stronger, $\tau_{U} \propto \frac{1}{\omega^{4}}$.

Considering that at low frequency this relation is in accordance with the well-known Klemens's expression [35, 37]

$$
\tau_{U}(\omega)=\frac{1}{B_{U} T \exp \left(-\frac{D}{T}\right)} \frac{1}{\omega^{2}},
$$


with $B_{U}=1.73 \times 10^{-19} \frac{s}{K}$ and $D=137.39 K$, we used Eq. (14) for the relaxation time at low frequency in $U$-processes.

Considering that the relaxation time has a stronger frequency dependency at higher frequencies [36], we employed a similar expression to the well-known Eq. (14), but with larger powers of frequencies to express the relaxation time at higher frequencies. We considered that the relaxation time follows

$$
\tau_{U}(\omega)=\left\{\begin{array}{ll}
\frac{1}{B_{U} T \exp \left(-\frac{D}{T}\right)} \frac{1}{\omega^{2}}, & \omega \leq \omega_{C} \\
\frac{1}{S_{U} T \exp \left(-\frac{D}{T}\right)} \frac{1}{\omega^{4}}, & \omega \geq \omega_{C}
\end{array},\right.
$$

where $\omega_{C}$ is the cross-over frequency. Here, $S_{U}$ is chosen such that we observe a continuous relaxation time at $\omega_{C}$,

$$
S_{U}=\frac{B_{U}}{\omega_{C}^{2}}
$$

The cross-over frequency, $\omega_{C}$, is then chosen to fit to the experimental data for thermal conductivity.

For the $N$-process we follow Ref. [38], and assume that the relaxation time is approximated as

$$
\tau_{N}(\omega)=\frac{1}{B_{N} T \omega^{2}}
$$

where $B_{N}$ can be used to adjust to the experimental data.

\section{SYSTEM OF MOMENTS}

\section{A. Moment equations}

Following Grad's moment method [23], we derive a set of macroscopic transport equations that can approximate the phonon-Boltzmann equation. For this means, we define the general macroscopic moment

$$
u_{<i_{1}, . . i_{n}>}^{\alpha}=\int_{B Z} \omega^{\alpha} n_{<i_{1} \ldots} n_{i_{n}>} f(\mathbf{x}, \mathbf{k}, t) d \mathbf{k},
$$

to relate the phonon properties to the macroscopic properties of the crystal. The indices in the angular brackets denotes the trace-free and symmetric part of the tensor. Our moment definition contains the powers of frequencies $\omega^{\alpha}$ with $\alpha=0,1,2, \ldots$, and the wave vector in the form of direction vectors, $n_{\left\langle i_{1} . . i_{n}\right\rangle}=\frac{k_{\left\langle i_{1} \ldots i_{n}\right\rangle}}{k^{n}}$.

Following Eq. (17), the energy density is obtained by

$$
\frac{e(\mathbf{x}, t)}{\hbar}=u_{0}^{1}(\mathbf{x}, t)
$$

The energy density flux and the momentum density of phonons will be related to the vectorial moments $u_{i}^{\alpha}$, by using the constitutive relations in the sequel.

In order to get a hierarchy of balance equations for the moments, we multiply the phonon-Boltzmann equation with the generic term

$$
\Upsilon_{i_{1}, . . i_{n}}^{\alpha}=\omega^{\alpha} n_{i_{1} \ldots} n_{i_{n}}
$$

and perform an integration over the $\mathbf{k}$-space in the Brillouin zone. This leads to a system of moment equations of the generic form

$$
\frac{\partial u_{i_{1}, . . i_{n}}^{\alpha}}{\partial t}+\frac{\partial F_{i_{1}, . . i_{n} i_{n_{p}}}^{\alpha}}{\partial x_{n_{p}}}=P_{i_{1}, . . i_{n}}^{\alpha}
$$

with the fluxes

$$
F_{i_{1}, \ldots i_{n} i_{n+1}}^{\alpha}=\int_{B Z} c_{i_{n+1}} \omega^{\alpha} n_{i_{1} \ldots} n_{i_{n}} f d \mathbf{k}
$$

and the productions

$$
P_{i_{1}, . . i_{n}}^{\alpha}=\int_{B Z} \omega^{\alpha} n_{i_{1} \ldots} n_{i_{n}} S(f) d \mathbf{k}
$$


For the scalar moments $u_{0}^{\alpha}$, we separate the equilibrium part from the non-equilibrium part, and define

$$
v_{0}^{\alpha}=u_{0}^{\alpha}-u_{0 \mid E}^{\alpha} .
$$

Here, the equilibrium part of the scalar moment is defined as

$$
u_{0 \mid E}^{\alpha}=\int_{B Z} \omega^{\alpha} f_{E} d \mathbf{k}
$$

Note that $F_{i_{1}, . . i_{n}}^{\alpha}$ has been defined as a rank $n$ tensor. It is helpful in the further calculation to decouple the trace and trace-free parts of tensors, and indicate the trace-free tensor by angled brackets around the indices. For example, for the second order tensor we can write

$$
F_{i j}^{\alpha}=F_{<i j>}^{\alpha}+\frac{1}{3} F_{0}^{\alpha} \delta_{i j}
$$

where $\delta_{i j}$ is the Kronecker delta function. Using similar tensorial calculation [25], we can decouple the trace and trace-free parts of a rank $n$ tensor. Therefore, the hierarchy of moment equations, using the trace-free symmetrical tensors, can be written as

$$
\begin{aligned}
& \frac{\partial u_{0}^{1}}{\partial t}+\frac{\partial F_{i}^{1}}{\partial x_{i}}=0 \\
& \frac{\partial v_{0}^{\alpha}}{\partial t}-\frac{\partial u_{0 \mid E}^{\alpha}}{\partial u_{0}^{1}} \frac{\partial F_{i}^{1}}{\partial x_{i}}+\frac{\partial F_{i}^{\alpha}}{\partial x_{i}}=P_{0}^{\alpha} \quad \alpha \geq 0, \neq 1 \\
& \frac{\partial u_{i}^{\alpha}}{\partial t}+\frac{\partial F_{<i j>}^{\alpha}}{\partial x_{j}}+\frac{1}{3} \frac{\partial F_{0}^{\alpha}}{\partial x_{i}}=P_{i}^{\alpha} \\
& \frac{\partial u_{<i j>}^{\alpha}}{\partial t}+\frac{\partial F_{<i j p>}^{\alpha}}{\partial x_{p}}+\frac{2}{5} \frac{\partial F_{<i}^{\alpha}}{\partial x_{j>}}=P_{<i j>}^{\alpha}, \\
& \frac{\partial u_{\left\langle i_{1} i_{2} \ldots i_{n}>\right.}^{\alpha}}{\partial t}+\frac{\partial F_{\left\langle i_{1} i_{2} \ldots i_{n} i_{n+1}>\right.}^{\alpha}}{\partial x_{n+1}}+\frac{n}{2 n+1} \frac{\partial F_{\left\langle i_{1} i_{2} \ldots i_{n-1}\right.}^{\alpha}}{\partial x_{i_{n}>}}=P_{<i_{1} i_{2} \ldots i_{n}>}^{\alpha} \quad n>2 .
\end{aligned}
$$

Note that based on the definition of moments, we have $u_{i_{1} i_{2} \ldots i_{n} k k}=u_{i_{1} i_{2} \ldots i_{n}}$. Moreover, $\alpha$ is the number of frequency powers in the non-equilibrium moments. The first equation in this hierarchy is the conservation of energy, where production term is zero in the right hand side.

\section{B. Closure for system of moments}

Taking moments of the phonon-Boltzmann equation generates a large system, consisting of an infinite number of balance equations (21). This system includes flux terms $F_{<i_{1} i_{2} \ldots i_{n} i_{n+1}>}^{\alpha}$, as well as production terms $P_{<i_{1} i_{2} \ldots i_{n}>}^{\alpha}$, that need to be determined to get a closed system of equations. The solution to this closure problem in kinetic theory, was first proposed by Grad [23], and then extended to the phonon kinetic theory in Ref. [6, 29]. Since flux and production terms are expressed through the distribution function, the closure problem is solved when we find the distribution function, that indeed depends on the moments. For this means, we follow the Grad's [23] idea to express the distribution function as a function of moments in Appendix A. Here, we present the final result as

$$
f_{G}=f_{E}+\frac{a^{3}}{32 \pi} \sum_{\substack{\eta, m \\ \neq E}} \frac{(2 m+1) ! !}{m !} \Phi^{\eta} X_{M}^{\eta+1}\left[\frac{u_{<j_{1}, . . j_{m}>}^{\eta}-u_{<j_{1}, . . j_{m}>E}^{\eta}}{\omega_{M}^{\eta}}\right] n_{<j_{1} \ldots} n_{j_{m}>}
$$

where $\omega_{M}=\frac{2 c_{0}}{a}$ is the reference frequency, and $X_{M}=\frac{\hbar \omega_{M}}{k_{B} T}$ is the non-dimensional inverse of temperature. For the detailed derivation of the distribution function, as well as the expressions for $\Phi^{\eta}$ see Appendix A.

\section{Flux terms}

Using the Grad distribution function, we can determine the fluxes as functions of moments. The fluxes have the general form

$$
F_{<i_{1} i_{2} \ldots i_{n} i_{n+1}>}^{\alpha}=\int_{B Z} c_{i_{n+1}} \omega^{\alpha} n_{<i_{1} \ldots} n_{i_{n}>} f_{G} d \mathbf{k}
$$


Substituting Eq. (22), and rearranging the equations gives

$$
F_{<i_{1} i_{2} . . i_{n}>}^{\alpha}=\left\{\begin{array}{ll}
c_{0} \omega_{M}^{\alpha}\left(A_{E}^{\alpha} \frac{u_{0 \mid E}^{\alpha}}{\omega_{M}^{\alpha}}+\sum_{\substack{\eta=0 \\
\neq 1}}^{n_{F}} A_{0}^{\alpha, \eta} \frac{v_{0}^{\eta}}{\omega_{M}^{\eta}}\right), & n=0 \\
c_{0} \omega_{M}^{\alpha}\left(\sum_{\eta=0}^{n_{F}} A_{n}^{\alpha, \eta} \frac{u_{<i_{1}, \ldots i_{n}>}^{\eta}}{\omega_{M}^{\eta}}\right), & n \neq 0
\end{array},\right.
$$

The detailed derivation of this relation, as well as the expressions for $A_{n}^{\alpha, \eta}$ and $A_{E}^{\alpha}$ can be found in Appendix B.

\section{Production terms}

Similar to the flux terms, we use the Grad distribution function to express the production terms as functions of moments. The production terms have the general form

$$
P_{<i_{1} i_{2} . . i_{n}>}^{\alpha}=\int_{B Z} \omega^{\alpha} n_{<i_{1} \ldots} n_{i_{n}>} S\left(f_{G}\right) d \mathbf{k}
$$

where $S\left(f_{G}\right)$ is obtained by using the Callaway model in Eq. (9). It is worth noting that by using the moment method $R$ and $N$-processes can be treated completely independently

$$
P_{<i_{1} i_{2} \ldots i_{n}>}^{\alpha}=P_{<i_{1} i_{2} \ldots i_{n}>, R}^{\alpha}+P_{<i_{1} i_{2} \ldots i_{n}>, N}^{\alpha} \text {. }
$$

a. Resistive process Considering the conservation of energy in the $R$-process Eq. (10), and expressing $f_{R}$ as a function of the non-equilibrium moments, we obtain the production moments for $R$-processes as

$$
P_{<i_{1} i_{2} \ldots i_{n}>, R}^{\alpha}=\left\{\begin{array}{ll}
-\frac{\omega_{M}^{\alpha}}{\tau_{R}^{0}} \sum_{\eta=0}^{n_{F}} J_{0, R}^{\alpha, \eta} \frac{v_{0}^{\eta}}{\omega_{M}^{\eta}} & n=0 \\
-\frac{\omega_{M}^{\alpha}}{\tau_{R}^{0}} \sum_{\eta=0}^{n_{F}} J_{n, R}^{\alpha, \eta} \frac{u_{<i_{1}, \ldots i_{n}>}^{\eta}}{\omega_{M}^{\eta}} & n \neq 0
\end{array},\right.
$$

Here, $\tau_{R}^{0}$ is the reference relaxation time for $R$-process that depends on the crystal, and will be discussed in the sequel. Note that we have $J_{0, R}^{1, \eta}=0$, which implies zero production in the case of energy moment, i.e., $n=0$ and $\alpha=1$. The detailed derivation of the above production matrix, as well as the expression for $J_{n, R}^{\alpha, \eta}$ can be found in Appendix C.

b. Normal process Using Eq. (11), we express $f_{N}$ as a function of non-equilibrium moments, and obtain the $N$-production moment as

$$
P_{<i_{1} i_{2} \ldots i_{n}>, N}^{\alpha}=\left\{\begin{array}{ll}
-\frac{\omega_{M}^{\alpha}}{\tau_{N}^{0}} \sum_{\eta=0}^{n_{F}} J_{0, N}^{\alpha, \eta} \frac{v_{0}^{\eta}}{\omega_{M}^{\eta}} & n=0 \\
-\frac{\omega_{M}^{\alpha}}{\tau_{N}^{0}} \sum_{\eta=0}^{n_{F}} J_{1, N}^{\alpha, \eta} \frac{u_{i}^{\eta}}{\omega_{M}^{\eta}} & n=1 . \\
-\frac{\omega_{M}^{\alpha}}{\tau_{N}^{0}} \sum_{\eta=0}^{n_{F}} J_{n, N}^{\alpha, \eta} \frac{u_{<i_{1} i_{2} \ldots i_{n}>}^{\eta}}{\omega_{M}^{\eta}} & n>1
\end{array} .\right.
$$

Here, $\tau_{N}^{0}$ is the reference relaxation time for $N$-process that also depends on the crystal, and will be discussed in the sequel. The detailed derivation of the above production matrix and an expression for $J_{n, N}^{\alpha, \eta}$ can be found in Appendix D.

\section{BOUNDARY CONDITIONS}

So far, we have derived the system of moments equations, and provided expressions for the flux and production terms to it. In this section we will derive the appropriate boundary conditions for this system of equations. 


\section{A. Microscopic model for the phonon-Boltzmann equation}

In order to describe the phonon-surface interactions we need a microscopic boundary condition that can be used to solve the phonon-Boltzmann equations. Later, this microscopic model will be utilized to obtain the macroscopic boundary conditions for the moment equations.

We use a phonon-surface model similar to the Maxwell boundary condition in classical theory [39], where the reflection kernel is a superposition of the kernels for diffusive reflection and specular reflection.

For phonons, we consider three possible scenarios when colliding with the surface [29, 40] :

- Thermalization: This process occurs when the impacting phonon with the surface is absorbed at the wall, and new phonons are emitted from the surface to the gas. The generated phonons will leave the surface at the equilibrium Bose distribution (5), with the temperature of the surface. In this process, impacting phonons exchange energy and momentum with the surface, which subsequently leads to an energy transfer across the crystal boundary.

- Specular reflection: In this process the energy and tangential momentum of the impacting phonons with the surface remain conserved, and only their normal momentums become inverted. This interaction does not lead to any energy transfer, or drag (like) forces across the crystal boundary.

- Isotropic scattering: This process accounts for the adiabatic interactions, while phonons transfer tangential momentum to the surface. The incoming phonons are reflected in a random direction, while keeping their impacting energy. This process is in analogy to the collision of a light gas particle to the heavy surface particle for classical gasses [41].

In order to describe the phonon-surface behavior we need the distribution function in an infinitesimal neighborhood of the wall, that we write as

$$
\bar{f}= \begin{cases}f^{*}(\mathbf{x}, \mathbf{k}, t), & n_{i} \nu_{i} \geq 0 \\ f(\mathbf{x}, \mathbf{k}, t), & n_{i} \nu_{i} \leq 0\end{cases}
$$

Here, $\nu_{i}$ is the surface normal unit vector, and $n_{i}=\frac{k_{i}}{k}$ is the unit phonon direction vector. The distribution function for phonons leaving the surface $\left(n_{i} \nu_{i} \geq 0\right)$ is expressed by $f^{*}$, and is described by the reflection model.

We define $\beta$ as the portion of phonons that are scattered or specularly reflected from the wall. Noting that the number of phonons are conserved in these two process, we assume that $\gamma$ of them are specularly reflected, and ( $1-\gamma)$ are isotropically scattered. Moreover, $\alpha$ is the relative amount of thermalized phonons. Thus, the distribution function for the particles leaving the surface reads

$$
f^{*}=\alpha f_{E}\left(T_{s}, k\right)+\beta \gamma f\left(k_{i}-2 k_{j} \nu_{j} \nu_{i}\right)+\beta \frac{(1-\gamma)}{\pi} \frac{1}{c} \int_{n_{k} \nu_{k}<0} c\left(-n_{k} \nu_{k}\right) f\left(k_{j}\right) d \Omega .
$$

Here, the first term in the right hand side denotes the thermalized phonons, which will be emitted with the equilibrium distribution function at the wall temperature, $T_{s}$. The second term describes the specular reflection of phonons, in which only the normal momentum will be inverted. The isotropic scattering of phonons, where phonons are reflected with a random angle, is described in the third term of Eq. (30).

This relation must also hold in the equilibrium state, when both incoming and outgoing particles follow the equilibrium distribution $f_{E}$ at $T=T_{s}$. Substituting this condition in Eq. (30) gives

$$
\alpha=(1-\beta) \text {. }
$$

Therefore, based on the crystal properties, this model for boundary condition introduces $\beta$ and $\gamma$ as the free parameters to fit to experimental data.

\section{B. Boundary conditions for moments}

To obtain the macroscopic boundary conditions for moments, we follow the ideas of Grad as outlined in [23, 42]. In this method, first we assume that the phonon distribution function can be approximated with the Grad distribution Eq. (22), and set $f=f_{G}$. Then, we require the continuity of certain normal fluxes over an infinitessimal surface element, that by using Eq. (29) gives

$$
\int \Upsilon_{A} n_{k} \nu_{k} \bar{f} d \mathbf{k}=\int \Upsilon_{A} n_{k} \nu_{k} f_{G} d \mathbf{k}
$$


where $\Upsilon_{A}$ is the polynomial of the frequency and the direction vector, that we already defined in Eq. (18). Following this method we obtain relations between the macroscopic moments and the wall properties, which shall be used as the boundary conditions for the macroscopic moments. Since $\bar{f}$ and $f_{G}$ agree for the incident particles, the above relation simplifies to

$$
\int_{n_{i} \nu_{i}>0} \omega^{\alpha} n_{i_{1} \ldots} n_{i_{n}} n_{k} \nu_{k} f^{*} d \mathbf{k}=\int_{n_{i} \nu_{i}>0} \omega^{\alpha} n_{i_{1} \ldots} n_{i_{n}} n_{k} \nu_{k} f_{G} d \mathbf{k}
$$

In order to perform the directional integration on the half space, it is best to use the normal-tangential frame with respect to the surface, where the phonon direction vector can be expressed as $n_{k}=\left\{\tau_{A}, \nu\right\}_{k}$. In this frame, the normal and tangential components to the surface can be written as

$$
\nu=n_{k} \nu_{k}=\cos \theta \quad \text { and } \quad \tau_{A}=\{\sin \theta \cos \phi, \sin \theta \sin \phi\}_{A}
$$

Using this notation, Eq. (32) simplifies to

$$
\int_{\nu>0} \omega^{\alpha} \tau_{A_{1}} \ldots \tau_{A_{r}} \nu^{n-r+1} f^{*} d \mathbf{k}=\int_{\nu>0} \omega^{\alpha} \tau_{A_{1}} \ldots \tau_{A_{r}} \nu^{n-r+1} f_{G} d \mathbf{k}
$$

where $r \leq n$. Grad [23] observed that in order to get a meaningful set of boundary conditions $\Upsilon_{A}$ must be even in the normal component of the directional vectors, hence $n-r$ must be even. Performing the integration in Eq. (32) leads to the general relation for the boundary conditions

$$
\begin{aligned}
\frac{32 \pi}{a^{3} X_{M}^{\alpha+1}}(1-\beta) & \left(\int_{B Z} X^{\alpha}\left(f_{E}-\overline{f_{E}}\left(T_{s}\right)\right) G(X) d X\right) \xi_{s}^{m} \delta_{\left\{B_{1} \ldots B_{s}\right\}} \\
-\beta(1-\gamma) \sum_{n} \mu^{\alpha} \frac{\left(u_{<\nu_{1} \ldots \nu_{n}>}^{\alpha}-u_{<\nu_{1} \ldots \nu_{n}>\mid E}^{\alpha}\right)}{\omega_{M}^{\alpha}} \xi_{s}^{m} \delta_{\left\{B_{1} \ldots B_{s}\right\}}=\sum_{n} \sum_{r=0}^{n} \frac{(2 n+1) ! !}{(n-r) ! r !}\left(\gamma \beta \sigma_{r+s}^{n+m}-\xi_{r+s}^{n+m}\right) & \frac{\left(u_{<A_{1} \ldots A_{r} \nu_{1} \ldots \nu_{n-r}>}^{\alpha}-u_{\left.<A_{1} \ldots A_{r} \nu_{1} \ldots \nu_{n-r}>\mid E\right)}^{\alpha} \delta_{\left\{A_{1} \ldots A_{r} B_{1} \ldots B_{s}\right\}}\right.}{\omega_{M}^{\alpha}}
\end{aligned}
$$

Here, $X=\frac{\hbar \omega}{k_{B} T}$ is the non-dimensional frequency. Moreover, the capital indices refer to the tangential, $\nu$ refers to the normal directions with respect to the surface, and $\delta_{\left\{B_{1} \ldots B_{s}\right\}}$ is the generalized tangential unit tensor. Note that the above boundary conditions are an extension to the simplified form of boundary equations presented in Ref. [29]. Details for derivation of this relation can be found in Appendix E and F .

\section{CLOSED SYSTEM OF MOMENT EQUATIONS AND BOUNDARY CONDITIONS}

\section{A. Macroscopic equations}

Substituting the description for the flux term Eq. (24), as well as the production terms Eqs. (27) and (28), provides a closed system of equations that can be solved to describe the thermal behavior of the crystal. We introduce the non-dimensional moments, time and space as

$$
U_{<i_{1} i_{2} \ldots i_{n}>}^{\alpha}=\frac{u_{<i_{1}, . . i_{n}>}^{\alpha}}{\omega_{M}^{\alpha}}, \quad \hat{t}=\frac{t c_{0}}{L}, \quad \hat{x}=\frac{x}{L},
$$

where $L$ is the length scale of the system. Based on the desired accuracy, we can use a specific number of transport equations for the direction and frequency. In the current study we employ three directional moments $\left(u_{0}^{\alpha}, u_{i}^{\alpha}, u_{<i j>}^{\alpha}\right)$. As a required condition, we will show that further increase in the number of directional moments, i.e., $u_{<i j k>}^{\alpha}, u_{<i j k l>}^{\alpha} \ldots$, will not cause any noticeable change in the solution. Considering three directional moments will lead to a set of $9 \times n_{F}$ macroscopic equations, where $n_{F}$ is the number of considered powers of frequency. By considering a small deviation from the equilibrium state, we can linearize the convection term in the non-equilibrium moments, and write Eq. (21) 
as

$$
\begin{aligned}
& \frac{\partial U_{0}^{1}}{\partial \hat{t}}+\sum_{\eta=0}^{n_{F}} A_{1}^{1, \eta} \frac{\partial U_{i}^{\eta}}{\partial \widehat{x}_{i}}=0 \\
& \frac{\partial V_{0}^{\alpha}}{\partial \hat{t}}+\sum_{\eta=0}^{n_{F}}\left(A_{1}^{\alpha, \eta}-\frac{\partial U_{0 \mid E}^{\alpha}}{\partial U_{0}^{1}} A_{1}^{1, \eta}\right) \frac{\partial U_{i}^{\eta}}{\partial \widehat{x}_{i}}=-\frac{1}{\operatorname{Kn}_{R}} \sum_{\substack{\eta=0 \\
\neq 1}}^{n_{F}} J_{0}^{\alpha, \eta} V_{0}^{\eta} \quad \alpha \geq 0, \neq 1, \\
& \frac{\partial U_{i}^{\alpha}}{\partial \hat{t}}+\sum_{\eta=0}^{n_{F}} A_{2}^{\alpha, \eta} \frac{\partial U_{<i j>}^{\eta}}{\partial \widehat{x}_{j}}+\frac{1}{3} \sum_{\substack{\eta=0 \\
\neq 1}}^{n_{F}} A_{0}^{\alpha, \eta} \frac{\partial V_{0}^{\eta}}{\partial \widehat{x}_{i}}+\frac{1}{3} \frac{\partial\left(A_{E}^{\alpha} U_{0 \mid E}^{\alpha}\right)}{\partial U_{0}^{1}} \frac{\partial U_{0}^{1}}{\partial \widehat{x}_{i}}=-\frac{1}{\mathrm{Kn}_{R}} \sum_{\eta=0}^{n_{F}} J_{1}^{\alpha, \eta} U_{i}^{\eta} \\
& \frac{\partial U_{<i j>}^{\alpha}}{\partial \widehat{t}}+\frac{2}{5} \sum_{\eta=0}^{n_{F}} A_{1}^{\alpha, \eta} \frac{\partial U_{<i}^{\eta}}{\partial \widehat{x}_{j>}}=-\frac{1}{\mathrm{Kn}_{R}} \sum_{\eta=0}^{n_{F}} J_{2}^{\alpha, \eta} U_{<i j>}^{\eta}
\end{aligned}
$$

Here, $\alpha$ is the number of frequency powers in the non-equilibrium moment, which can be up to $n_{F}$. Moreover,

$$
J_{n}^{\alpha, \eta}=J_{n, R}^{\alpha, \eta}+\frac{\mathrm{Kn}_{R}}{\mathrm{Kn}_{N}} J_{n, N}^{\alpha, \eta}
$$

and

$$
\mathrm{Kn}_{R}=\frac{c_{0} \tau_{R}^{0}}{L} \quad \text { and } \quad \mathrm{Kn}_{N}=\frac{c_{0} \tau_{N}^{0}}{L},
$$

are the reference Knudsen numbers for the $R$ and $N$-processes. The temperature and heat flux implicitly appear in the moments equations, and will be discussed in details in the sequel.

The boundary conditions for this system, are obtained from Eq. (34) as

$$
\begin{gathered}
U_{\nu}^{\alpha}=-\frac{(1-\beta)}{2(\beta+1)} \frac{\Delta T}{T_{S}} Y^{\alpha}(T) U_{0}^{1}-\frac{(1-\beta)}{2(\beta+1)} V_{0}^{\alpha}-\frac{15}{16} \frac{(1-\beta)}{(\beta+1)} U_{<\nu \nu>}^{\alpha} \quad \alpha \geq 0, \neq 1, \\
U_{<A_{1} \nu>}^{\alpha}=-\frac{3}{8} \frac{(1-\gamma \beta)}{(\gamma \beta+1)} U_{A_{1}}^{\alpha} \quad \alpha \geq 0, \neq 1 .
\end{gathered}
$$

Here, we linearized the first term of Eq. (34) in the temperature difference $\Delta T=T_{S}-T$, to get

$$
Y^{\alpha}(T)=\frac{\int_{B Z} \frac{X^{\alpha+1} \exp (X) G(X)}{(\exp (X)-1)^{2}} d X}{X_{M}^{\alpha-1} \int_{B Z} \frac{X G(X)}{\exp (X)-1} d X} .
$$

The first equation in the system of moments (36) represents the transport equation for the energy moment, with no production on the right hand side, and will be discussed in the next section. The transport equations for the non-equilibrium scalar moments and vectorial moments are shown in the second and third equations (36).

The vectorial moments will be related to the phonon momentum using the constitutive relations. The transport equations for the vectorial moments include second-rank tensorial moments $U_{<i j>}^{\eta}$, that can be related to the phonon stress. Dreyer and Struchtrup [6] showed that using the system of moment including the phonon stress can successfully express the second sound in the crystal. Therefore, the system of moments (36) are expected to include the corresponding terms for the second sound.

\section{B. Energy, temperature and heat flux}

The energy moment $U_{0}^{1}$ appearing in the system of equations (36) varies with temperature as

$$
U_{0}^{1}=\frac{4 \pi k_{B}^{4} y}{\hbar^{4} c_{0}^{3} \omega_{M}}\left(\int_{B Z} \frac{X_{M}^{2} X G(X)}{\exp (X)-1} d X\right) T^{4} .
$$


Note that in the above equation $X$ and $X_{M}$ are also a function of temperature. In the case of linear dispersion relation with infinite Brillouin zone, i.e., Debye assumptions [4], this relation simplifies to

$$
U_{0_{\mid D}}^{1}=\frac{4 \pi^{5} k_{B}^{4} y}{15 \hbar^{4} c_{0}^{3} \omega_{M}} T^{4} .
$$

However, this assumption is only valid at low temperatures. Variation of the energy moment relative to the Debye energy moment with respect to the temperature is shown in Fig. 2. It is observed that at room temperature the energy moment deviates from the Debye energy moment, which reiterates the limitation of the Debye assumptions.

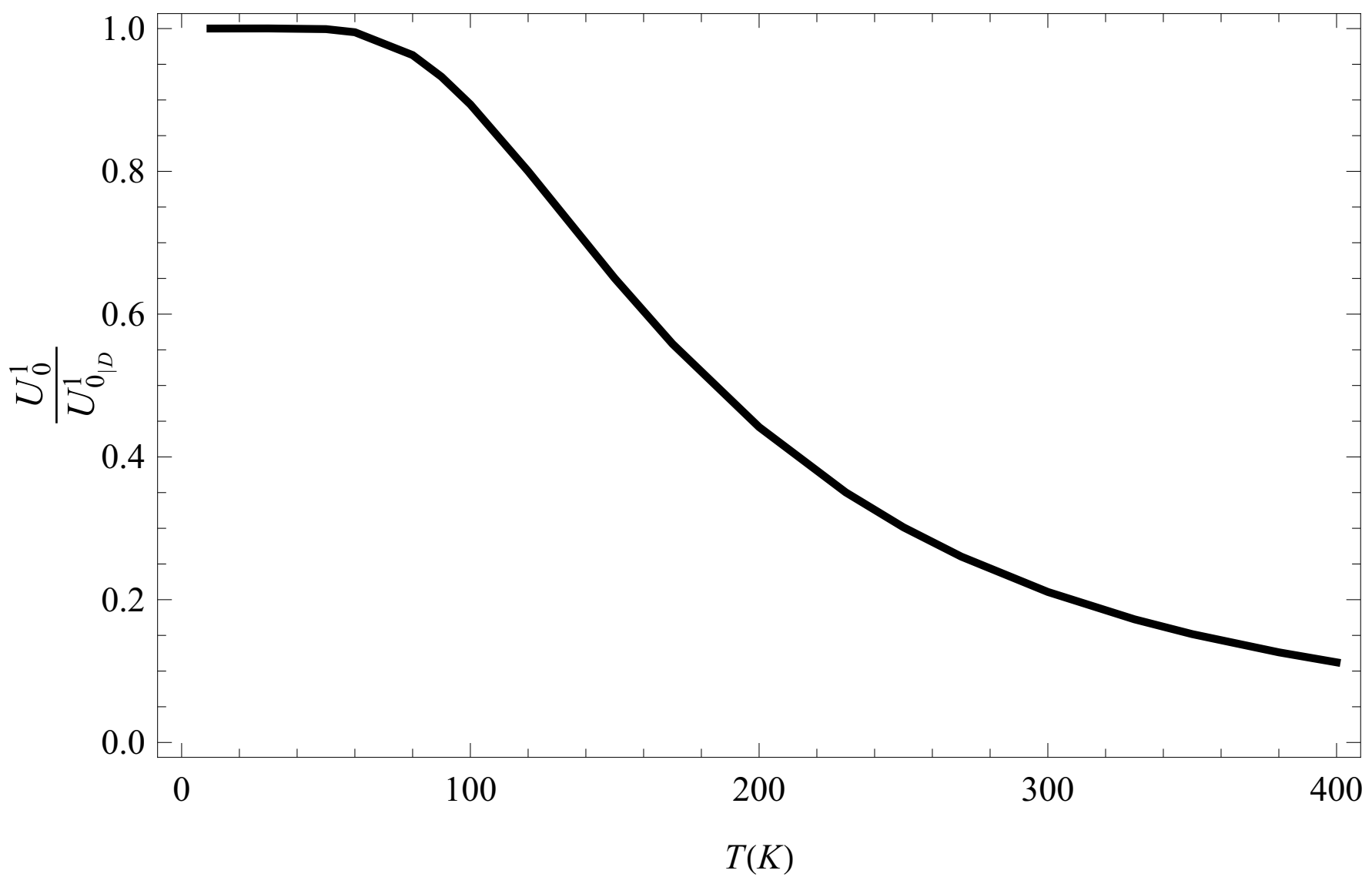

FIG. 2. Variation of the energy moment relative to the Debye energy moment with respect to the temperature for Silicon

Another look into Eq. (36) shows that energy flux appears as a summation over the vectorial moments

$$
Q_{i}=\sum_{\eta=0}^{n_{F}} A_{1}^{1, \eta} U_{i}^{\eta} .
$$

Note that the non-dimensional heat flux $Q_{i}$, relates to the dimensional heat flux $q_{i}$ as

$$
Q_{i}=\frac{q_{i}}{c_{o}\left(\hbar \omega_{M}\right)}
$$

We use Eq. (38) to obtain the energy flux as a function of energy gradient, and derive the thermal diffusivity.

\section{Thermal diffusivity and thermal conductivity}

The thermal diffusivity, $\varkappa$ relates the heat flux to the gradient of energy as

$$
q_{i}=-\varkappa \frac{\partial u_{0}^{1}}{\partial x_{i}} .
$$


Equation (36)-3 shows the dependency of the vectorial moments on the gradient of energy moment, that will be used to obtain the thermal diffusivity. Considering that Fourier's law Eq. (39), is valid in the first order of Kn, we perform a Chapman-Enskog (CE) expansion [16] on the system of moments, to identify the first order terms contributing to the Fourier's heat flux.

We expand the non-equilibrium moments in the smallness parameter $\mathrm{Kn}_{R}$ as

$$
\begin{aligned}
V_{0}^{\alpha} & =V_{0,0}^{\alpha}+\mathrm{Kn}_{R} V_{0,1}^{\alpha}+O\left(\mathrm{Kn}_{R}^{2}\right), \\
U_{i}^{\alpha} & =U_{i, 0}^{\alpha}+\mathrm{Kn}_{R} U_{i, 1}^{\alpha}+O\left(\mathrm{Kn}_{R}^{2}\right), \\
U_{<i j>}^{\alpha} & =U_{<i j>, 0}^{\alpha}+\mathrm{Kn}_{R} U_{<i j>, 1}^{\alpha}+O\left(\mathrm{Kn}_{R}^{2}\right) .
\end{aligned}
$$

Then, we substitute this description in the system of moments. For example, Eq. (36)-2 reads

$$
\begin{array}{r}
\frac{\partial\left(V_{0,0}^{\alpha}+\mathrm{Kn}_{R} V_{0,1}^{\alpha}+O\left(\mathrm{Kn}_{R}^{2}\right)\right)}{\partial \hat{t}}+\sum_{\eta=0}^{n_{F}}\left(A_{1}^{\alpha, \eta}-\frac{d U_{0 \mid E}^{\alpha}}{d U_{0}^{1}} A_{1}^{1, \eta}\right) \frac{\partial\left(U_{i, 0}^{\eta}+\mathrm{Kn}_{R} U_{i, 1}^{\eta}+O\left(\mathrm{Kn}_{R}^{2}\right)\right)}{\partial \hat{x}_{i}}= \\
-\sum_{\eta=0}^{n_{F}} \frac{1}{\mathrm{Kn}_{R}} J_{0}^{\alpha, \eta}\left(V_{0,0}^{\eta}+\mathrm{Kn}_{R} V_{0,1}^{\eta}+O\left(\mathrm{Kn}_{R}^{2}\right)\right)
\end{array}
$$

Collecting the powers of $\mathrm{Kn}_{R}^{-1}$ in the system of moments gives

$$
V_{0,0}^{\alpha}=U_{i, 0}^{\alpha}=U_{<i j>, 0}^{\alpha}=0,
$$

that implies non of the above moments have zeroth order contributions. Applying the CE expansion to (36)-3 with the updated form of (40), and collecting the contribution of order $\mathrm{Kn}_{R}^{0}$ gives

$$
\frac{1}{3} \frac{\partial\left(A_{E}^{\alpha} U_{0 \mid E}^{\alpha}\right)}{\partial U_{0}^{1}} \frac{\partial U_{0}^{1}}{\partial \widehat{x}_{i}}=-\frac{1}{\mathrm{Kn}_{R}} \sum_{\eta=0}^{n_{F}} J_{1}^{\alpha, \eta} U_{i, 1}^{\eta} .
$$

Using the description of energy flux from (38), and the first order contribution of the vectorial moments (41), the thermal diffusivity can be written as

$$
\varkappa=\varkappa^{0} \sum_{\eta, \gamma=0}^{n_{F}} A_{1}^{1, \eta}\left(\left(J_{1}^{-1}\right)^{\eta \gamma} \frac{\partial\left(A_{E}^{\gamma} U_{0 \mid E}^{\gamma}\right)}{\partial U_{0}^{1}}\right)
$$

where

$$
\varkappa^{0}=\frac{1}{3} \tau_{R}^{0} c_{0}^{2}
$$

is the reference thermal diffusivity, obtained by considering a constant relaxation time at the reference temperature.

Equation (42) shows that the thermal diffusivity predicted by the system of moments is a correction to the reference value, due to the frequency dependency in the relaxation time, i.e., the contribution of the production term $J_{1}^{\eta \gamma}$. It has been shown in Ref. [38] that the correction resulting from the Normal scattering process can be disregarded in the case of Silicon. Therefore, in order to adjust our relaxation time we assume that we only have $R$-process in our system, and neglect the $N$-process contribution.

Figure 3 shows the variation of the thermal conductivity with the temperature. Thermal conductivity, $\kappa$ is related to the thermal diffusivity as

$$
\kappa=\varkappa \rho C
$$

where $\rho$ is the mass density of the solid, and $C$ is the specific heat obtained in Eq. (8). We considered 6 powers of frequency in our system, i.e., $n_{F}=6$. In order to fit to the experimental data for thermal conductivity, we chose the cross-over between the two relaxation times in Eq. (15) to occur at 0.3 of the length of Brillouin zone, i.e., $\frac{\frac{h \omega_{C}}{k_{B} T}}{B Z}$ $=0.3$. Note that since $B Z$ in this expression also depends on the temperature, the considered value for the cross-over frequency does not vary with the working temperature. The predicted results from the system of moments are in very good agreement with the experimental values for the Silicon. We could extend the comparison of the thermal 


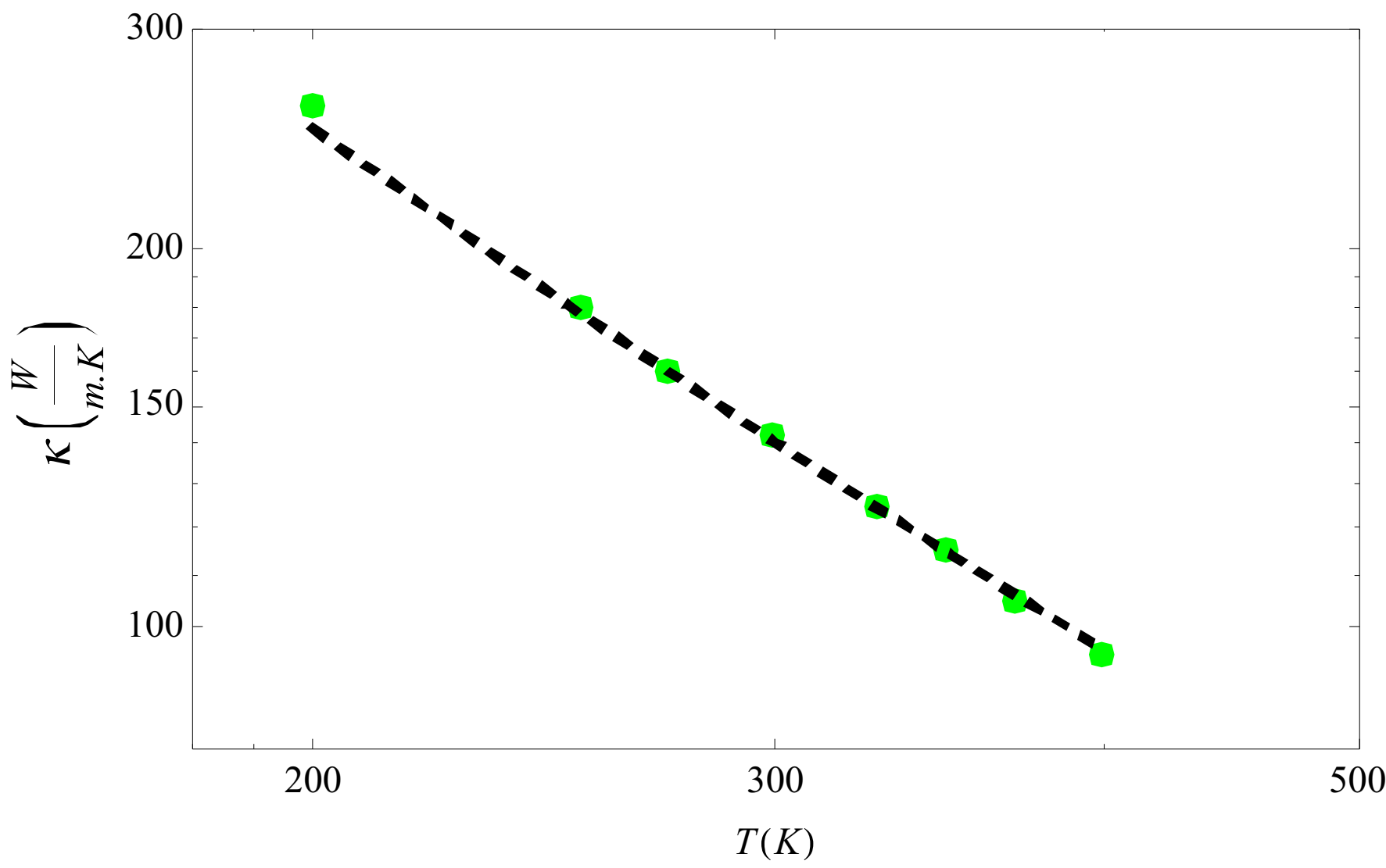

FIG. 3. Variation of the thermal conductivity with the temperature for Silicon, line: system of moments, dots: experimental data in Ref. [4]

conductivity to larger temperatures, however, we are interested in the room temperature comparison in the current study.

The effect of increasing the number of frequency powers on the thermal conductivity is depicted in Fig. 4 . Here we show the relative changes in the thermal conductivity $\phi_{n_{F}}=\frac{\left|\kappa_{n_{F}}-\kappa_{n_{F}-1}\right|}{\kappa_{n_{F}}}$ with successive increase in the number of considered frequencies, $n_{F}$ at $T=300 \mathrm{~K}$. We observed that the predicted results for thermal conductivity do not change noticeably by increasing the number of frequency powers to more than 6 . Thus, we set $n_{F}=6$ for the following results in this study.

\section{ANALYTICAL SOLUTION IN SIMPLE GEOMETRIES}

The system of moment equations (36) equipped with the boundary conditions (37) are a set of linear partial differential equations that can be analytically solved in simple geometries. First we study the behavior of onedimensional wave obtained by system of moments, and then we investigate the one-dimensional Poiseuille flow for the phonon gas.

\section{A. 1-D Heat conduction with periodic initial condition}

As the first application we look into the decay of energy amplitude with time in a one-dimensional body with periodic initial conditions such as Fig. 5. Nelson et al. [30] experimentally studied this problem by interfering two laser beams, and exposing a wafer to the diffraction pattern. In this experiment, a sinusoidal energy pattern was initialized in a thin silicon wafer at room temperature, and the thermal decay was measured to determine a wavelength dependency of the damping coefficient. They observed that at room temperature, the thermal transport in Silicon significantly deviates from the diffusion model already at micron distances. More specifically, they showed that low 


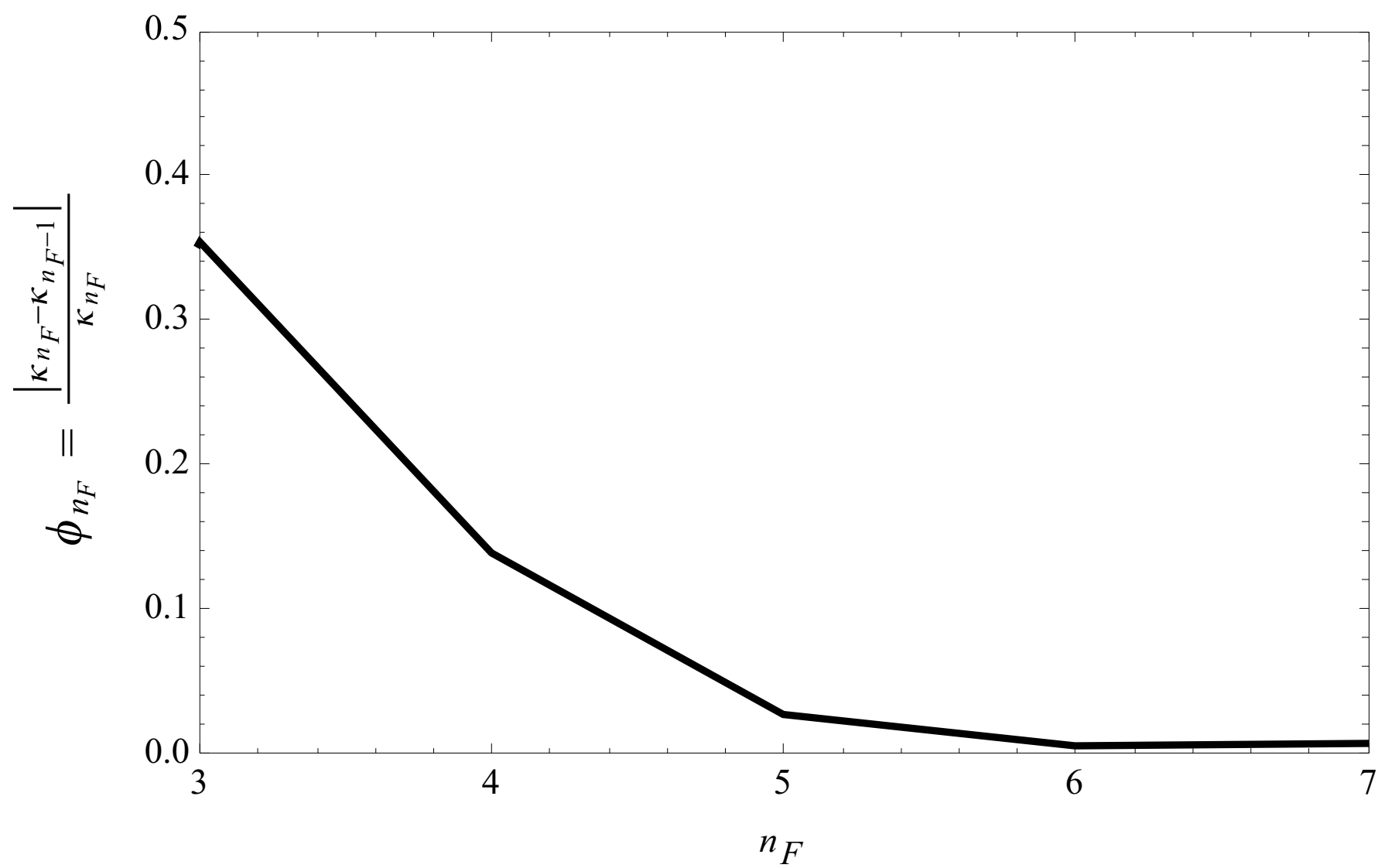

FIG. 4. Variation of the relative change in the thermal conductivity with the number of considered frequency powers.

frequency phonons, with rather large mean free paths that cannot be described by the diffusion model, significantly contribute to the heat transfer at micron distances.

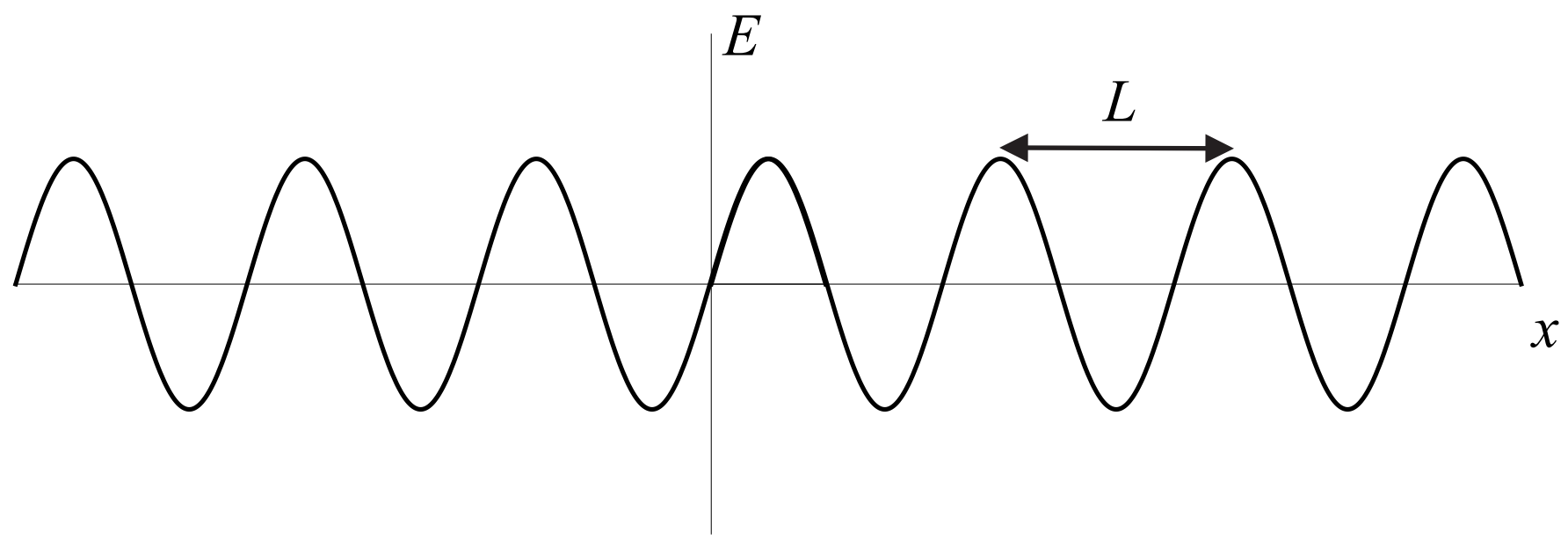

FIG. 5. One-dimensional body with periodic initial condition

Maznev et al. [43] investigated this problem by proposing a model in which the high-frequency phonons are described by the thermal diffusion equation, while the low-frequency phonons are described by the phonon-Boltzmann equation. They reported good agreement with the experimental data.

We use the moment method to predict the damping behavior as a function of the grating's wavelength, and compare it with the data reported in Ref. [43]. Due to the periodic nature of this problem, we do not need to use boundary conditions at both ends of the specimen, and will study the flow behavior in the bulk. 
The depth of wafer used in Ref. [30] was much smaller than its length and width, such that the sinusoidal pattern remains homogenous throughout the entire specimen. Moreover, Nelson et al. used wafers with different thickness to study the effect of thickness (boundary conditions) on the heat flow. For simple analysis, we only consider a one-dimensional flow with infinite thickness to neglect effects of boundaries. Then, we validate our numerical results with the reported data in Ref. [30] for the infinite thickness.

\section{System of moment solution}

In one-dimension, we can write the linear system (36) as

$$
\frac{\partial U_{A}}{\partial t}+A_{A B} \frac{\partial U_{B}}{\partial x}=-C_{A B} U_{B}
$$

where $A_{A B}$ and $C_{A B}$ correspond to the variable vector

$$
U=\left\{U_{0}^{1}, V_{0}^{\alpha}, U_{i}^{\alpha}, U_{<i j>}^{\alpha}\right\} \alpha=0,1, \ldots, n_{F}
$$

Here, $\alpha$ is the number of frequency powers in the non-equilibrium moment, which can be up to $n_{F}$. Considering the periodic nature of the problem, we make the harmonic wave ansatz

$$
U_{A}(x, t)=\widetilde{U}_{A} \exp (i(\digamma x-\Omega t))
$$

where $\widetilde{U}_{A}$ is the complex amplitude, $\digamma$ and $\Omega$ are, respectively, wavenumber and frequency of the harmonic wave. By inserting this relation in Eq. (43) we get the algebraic equation

$$
\left(i \Omega \delta_{A B}-i \digamma A_{A B}+C_{A B}\right) U_{B}=0
$$

that only has non-trivial solutions when the determinant of the matrix inside the parenthesis becomes zero. This will lead to an eigenvalue problem, where $\Omega$ is the vector of eigenvalues for the matrix of $\digamma A_{A B}+i C_{A B}$. For each eigenvalue there is a specific solution to the wave ansatz. By considering that only the energy moment was initially non-zero and equal to $\operatorname{Exp}(i \digamma x)$, the general solution can be obtained by adding all individual solutions together to form

$$
U_{A}(x, t)=\mathrm{E} \sum_{B} Q_{A B} Q_{B 1}^{-1} \exp \left(i\left(\digamma x-\Omega_{B} t\right)\right)
$$

Here, $Q_{A B}$ is the matrix of eigenvectors. We use our MATHEMATICA code to obtain the solution for the above expression. Note that the solution can be generated for an arbitrary number of moments, by simply expanding the dimensions of $U_{A}, A_{A B}$ and $C_{A B}$.

\section{Fourier's law solution}

The linearized heat equation in one-dimension reads

$$
\frac{\partial U_{0}^{1}}{\partial t}-\varkappa \frac{\partial^{2} U_{0}^{1}}{\partial x^{2}}=0 .
$$

Using the wave ansatz leads to a quadratic relation between the frequency and the wavenumber

$$
\Omega=-i \varkappa \digamma^{2}
$$

that is the case when diffusion is the dominant energy transfer mechanism. Using the Fourier law to solve the one-dimensional heat conduction problem gives

$$
U_{0}^{1}=\mathrm{E} \exp \left(-\varkappa \digamma^{2} t\right) \cos (\digamma x) .
$$

This equation shows that the Fourier law predicts a pure exponential decay for the energy moment by time. This exponential decay depends on the thermal diffusivity and the initial wavevector. 
Nelson et al. [30] conducted the thermal decay experiment on 15 transient gradient periods ranging from 2.4 to $25 \mu \mathrm{m}$, and reported that within the whole range of grating periods the thermal decay remains exponential.

First, we use the system of moments to investigate if the energy moment follows an exponential decay. For this means, first we assume that the energy moment follows an exponential function such as Eq. (45), however, the coefficient in the exponential argument is not necessarily the thermal diffusivity $\varkappa$. Then, we consider $t_{1}$ and $t_{2}$ as two arbitrary times during the decay, and plot $-\log \frac{U_{0}^{1}}{\mathrm{E}}$ as a function of $\digamma^{2} t$ in this period. This curve is compared with the predicted results from Eq. (44) for different values of grating period in Fig. 6.

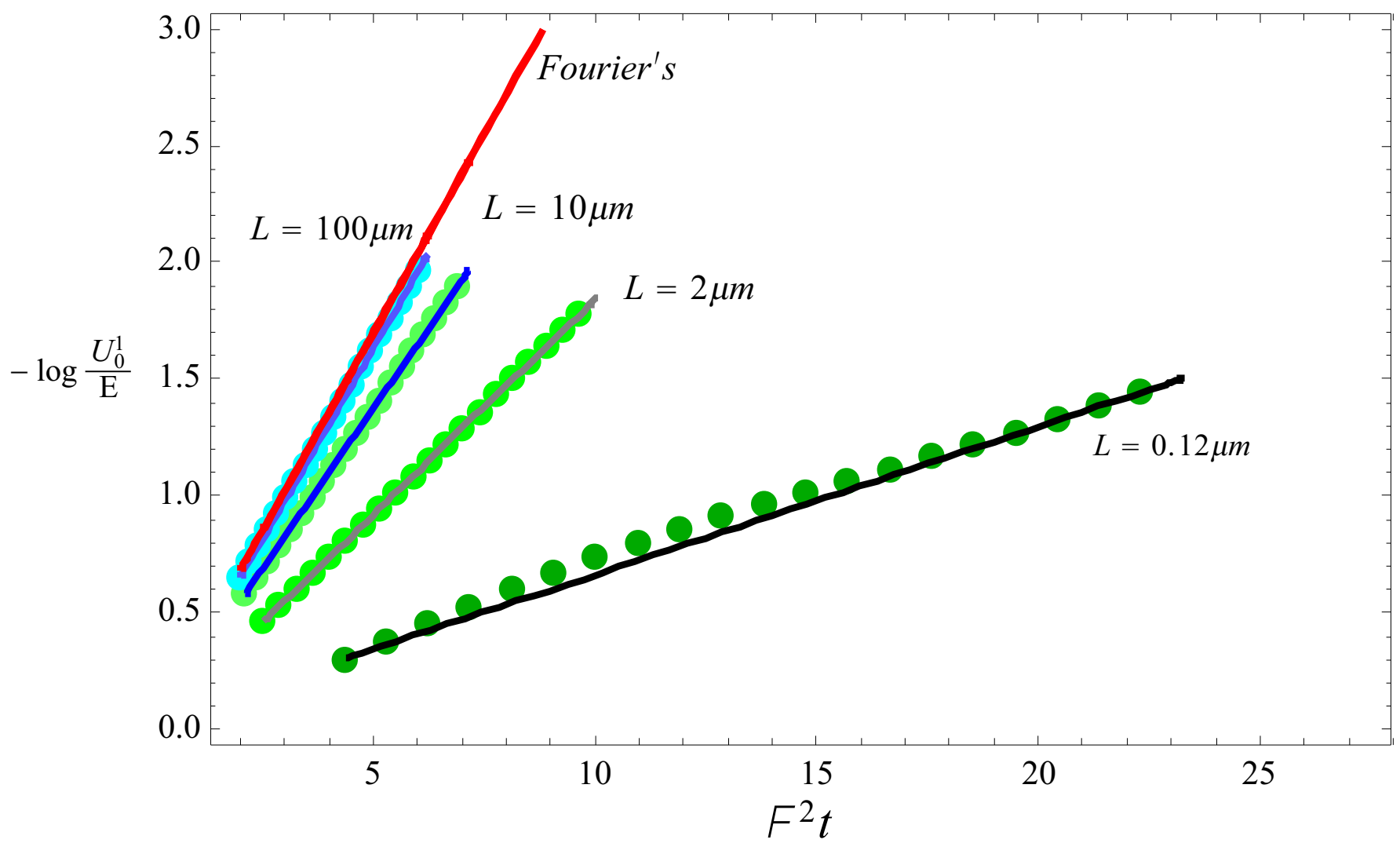

FIG. 6. Variation of the energy decay with time for various grating periods, Markers: solution to Eq. (44), Lines: exponential function passing through $t_{1}$ and $t_{2}$, Red line: Fourier's solution in Eq. (45)

For $t_{1}$ and $t_{2}$ we obtained the required time that the predicted energy by the system of moments drops to $80 \%$ and $20 \%$ of its initial value, respectively. We observed that as long as the grating period remains larger than $0.5 \mu m$, the decay follows a pure exponential curve, which is in accordance with the reported results in Ref. [30]. Moreover, it is observed that by increasing the grating length, the solution of system of moments approaches the Fourier's law solution, which is depicted by the red line.

Considering that the decay curve remains exponential for $L>0.5 \mu \mathrm{m}$, we define the decay parameter

$$
\Gamma\left(\digamma^{2}\right)=\frac{\ln \left(\frac{U_{0}^{1}\left(0, t_{1}\right)}{U_{0}^{1}\left(0, t_{2}\right)}\right)}{t_{2}-t_{1}} .
$$

Note that as long as we have an exponential decay, the choice of $t_{1}$ and $t_{2}$ are arbitrary, and will not change the value of the decay parameter. For the Fourier law we have $\Gamma_{F}=\varkappa \digamma^{2}$, that shows a quadratic decay with the wavenumber.

Figure 7 shows the decay parameter $\Gamma$ relative to the bulk Fourier decay as a function of the grating period for the moment solutions and the reported data in Ref. [30]. Nelson et al. [30] employed an effective thermal conductivity model, that takes into account the diffusion and ballistic transport of phonons, to report the energy decay when boundary scattering is not present. 
For our numerical results we employed three forms of relaxation times: the cross-over relaxation time model Eq. (15) depicted by the black curve, Klemens model Eq. (14) depicted by the blue curve, and the constant relaxation time model as shown by the red curve. Although all employed relaxation time models predict the exact thermal conductivity (first order contribution) at this temperature, this figure demonstrates their short-coming in capturing higher order non-equilibrium phenomenon, and suggests the importance of employing appropriate relaxation times in the Callaway model.

We adjusted the free parameter in the relaxation time for the N-process Eq. (16), to get the best agreement with reported data. It is seen that at larger grating period the decay parameter approaches to unity, which shows the domination of diffusion in the heat transport mechanisms. It is observed that employing the appropriate relaxation time in the system of moments provides very good agreement with the reported results in Ref. [30].

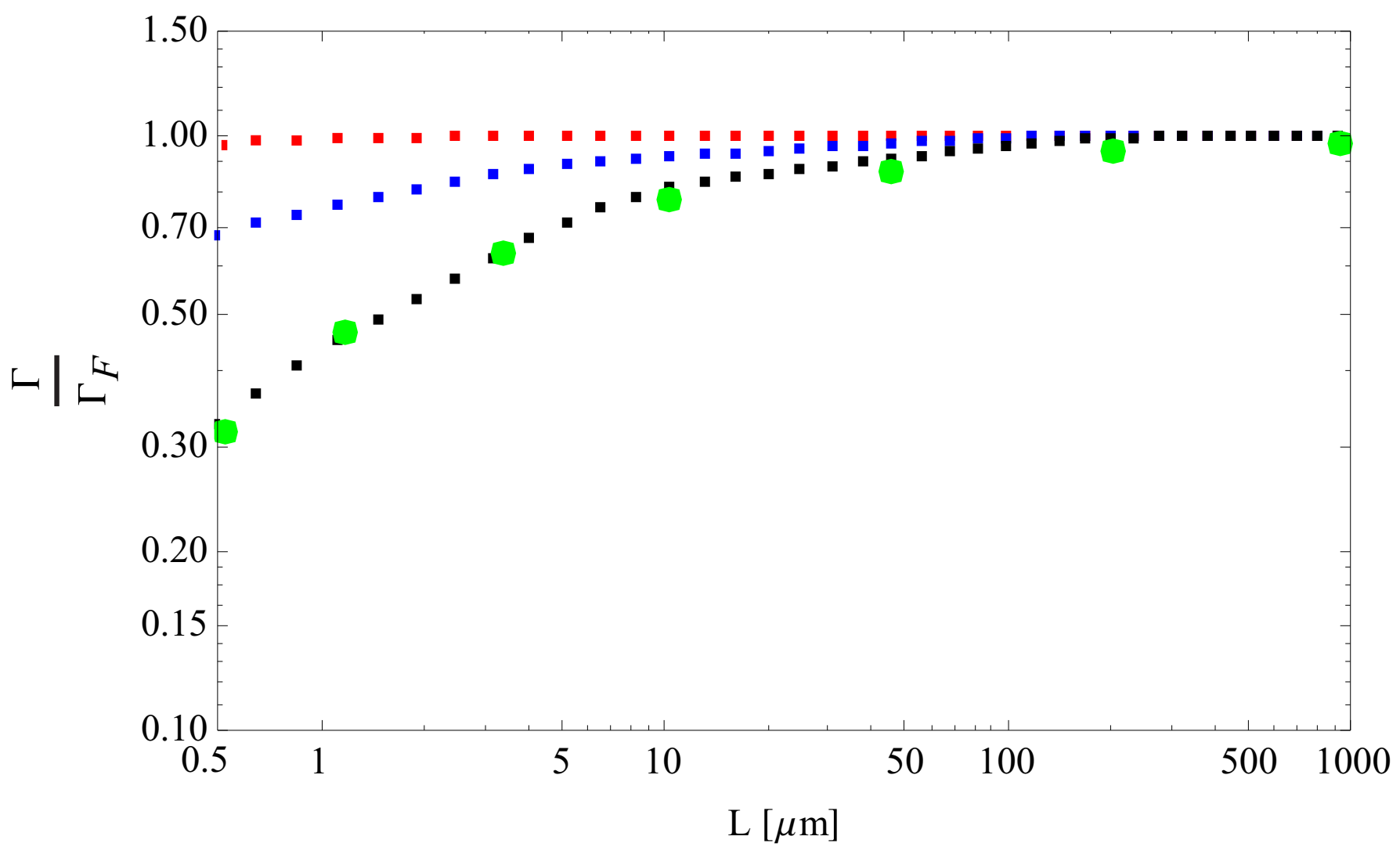

FIG. 7. Variation of the decay parameter relative to the bulk Fourier decay with the grating period, green: reported data in Ref. [30], black: cross-over model, blue: Klemens model, red: constant relaxation time model

In order to ensure the independency of the solution on the number of moments, we increase the number of directional moments $n_{D}$, and compared the relative deviation from the most accurate solution. Figure 8-a shows the variation of $\Phi_{n_{D}}=\frac{\left|\Gamma_{n_{D}}-\Gamma_{S}\right|}{\Gamma_{n_{D}}}$ with the number of considered directional moments, where $\Gamma_{S}$ is the solution of our largest systems with $n_{F}=n_{D}=7$. The comparison is conducted for 3 grating periods, $L=1.5 \mu m$ (square), $L=2.5 \mu m$ (circle) and $L=10 \mu \mathrm{m}$ (triangle). It is observed that by considering 3 directional moments, the relative deviation from the solution of large system will be around $1 \%$, and the solution is more or less converged. This system, that includes second rank tensorial moment, is rather simple for our analytical calculation, and will be used in the sequel.

Figure 8-b shows the energy decay traces for grating periods from 3.2 to $18 \mu \mathrm{m}$. This figure corresponds to the experimental results of Fig. 2 in Ref. [30]. It is seen that the thermal decay becomes slower at larger grating period. At larger grating period, it takes longer for heat to move from the peak to the null.

Note that in the experimental results of Ref. [30] the scattering of the phonons at the boundaries reduces the thermal conductivity (thickness effects). However, for our simple analytical calculation we assumed that the silicon specimen is wide enough that the boundary conditions do not play a role in the solution. The obtained results from the system of moments in Fig. 8-b are qualitatively similar to the experimental data of Fig. 2 in Ref. [30]. 


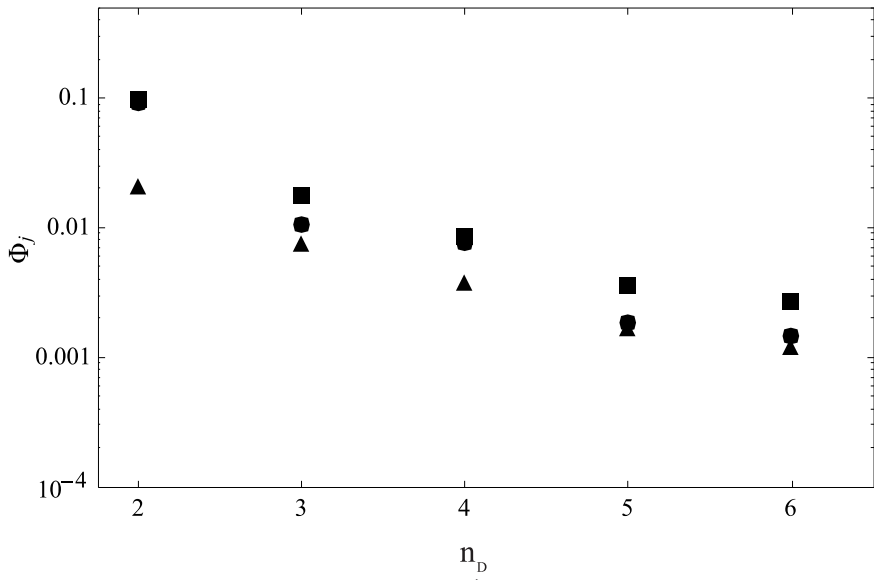

a)

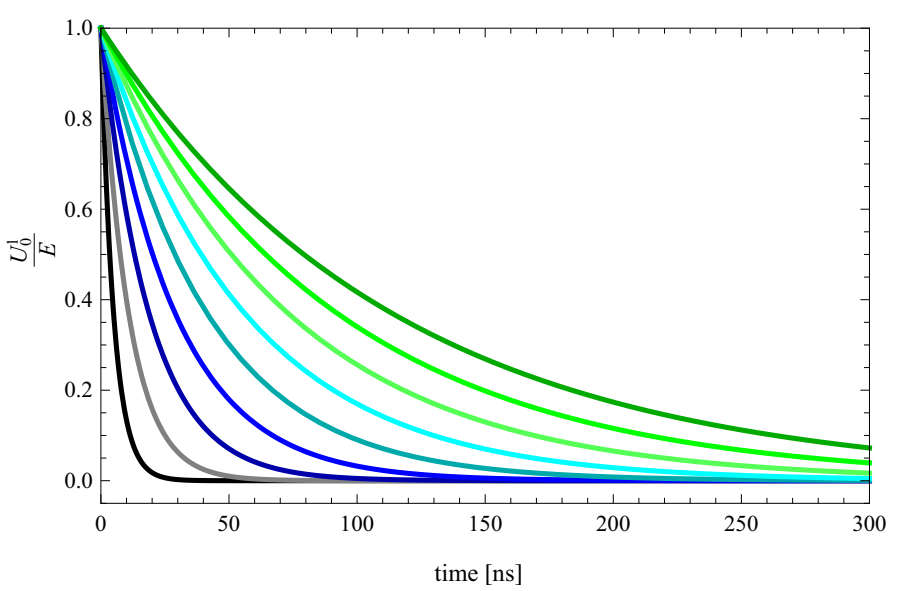

b)

FIG. 8. a) Relative changes in thermal decay parameter with the successive increase in the number of directional moments for $L=1.5 \mu \mathrm{m}$ (squar), $L=2.5 \mu \mathrm{m}$ (circle) and $L=10 \mu \mathrm{m}$ (triangle). b) Energy decay for grating periods of $L=2.3$ to $18 \mu \mathrm{m}$, corresponding to the reported results in Ref. [30].

\section{B. 1-D Poiseuille flow of phonons}

As another application we use the system of moments to solve one-dimensional Poiseuille flow of phonons in a heat conductor of thickness $2 L$, as depicted in Fig. 9. We assume that there is a constant energy gradient of $\frac{\partial U_{0}^{1}}{\partial x}$ along the $x$-direction inside a semi-infinite silicon specimen, with adiabatic boundaries at $y= \pm L$. This problem is analogous to the one-dimensional pressure driven flow in fluid dynamics, where the pressure gradient in the fluid is replaced by energy gradients inside the solid.

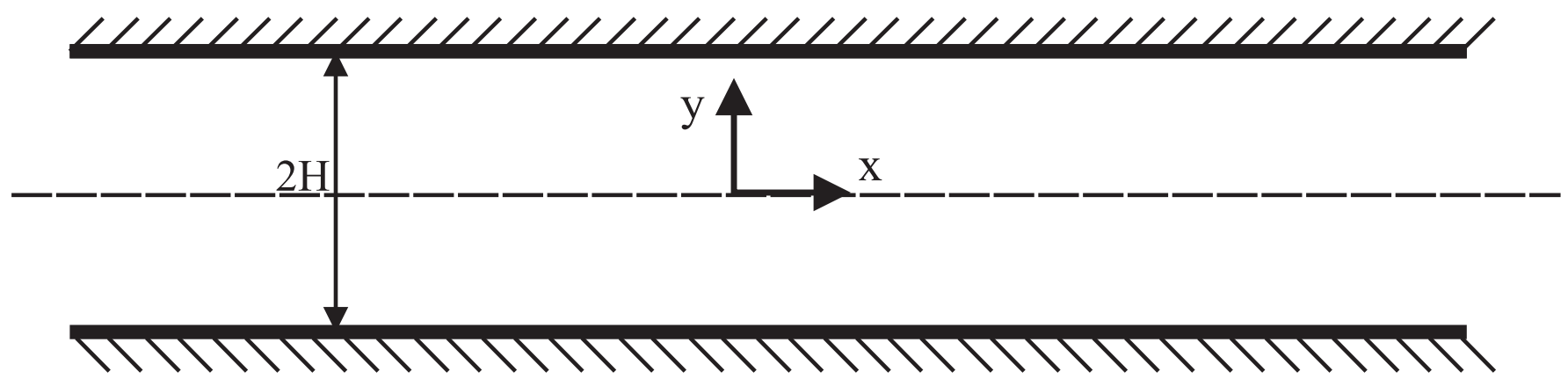

FIG. 9. One-dimensional Poiseuille flow

As the first step to solve Poiseuille flow, we replace one of the vectorial moments with the energy flux $Q_{i}$, to have an explicit relation for energy flux in our equations. By this change of variable, providing the solution becomes more straightforward. Using the description for energy flux from Eq. (38) we replace $U_{i}^{1}$ as

$$
U_{i}^{1}=\frac{1}{A_{1}^{1,1}} Q_{i}-\sum_{\substack{\eta=0 \\ \eta \neq 1}}^{n_{F}} \frac{A_{1}^{1, \eta}}{A_{1}^{1,1}} U_{i}^{\eta}
$$

By considering the steady state condition, where all moments except for energy have zero gradient in $x$-direction, 
we can decouple Eq. (36) to get

$$
\begin{aligned}
& \frac{\partial Q_{y}}{\partial \widehat{y}}=0, \\
& \sum_{\eta=0}^{n_{F}} A_{2}^{\alpha, \eta} \frac{\partial U_{<x y>}^{\eta}}{\partial \widehat{y}}+\frac{1}{3} \frac{\partial\left(A_{E}^{\alpha} U_{0 \mid E}^{\alpha}\right)}{\partial U_{0}^{1}} \frac{\partial U_{0}^{1}}{\partial \widehat{x}}=-\frac{1}{\operatorname{Kn}_{R}} \frac{J_{1}^{\alpha, 1}}{A_{1}^{1,1}} Q_{x}-\frac{1}{\operatorname{Kn}_{R}} \sum_{\substack{\eta=0 \\
\eta \neq 1}}^{n_{F}}\left(J_{1}^{\alpha, \eta}-J_{1}^{\alpha, 1} \frac{A_{1}^{1, \eta}}{A_{1}^{1,1}}\right) U_{x}^{\eta}, \\
& \frac{1}{5} \frac{A_{1}^{\alpha, 1}}{A_{1}^{1,1}} \frac{\partial Q_{x}}{\partial \widehat{y}}+\frac{1}{5} \sum_{\substack{\eta=0 \\
\eta \neq 1}}^{n_{F}}\left(A_{1}^{\alpha, \eta}-\frac{A_{1}^{\alpha, 1}}{A_{1}^{1,1}} A_{1}^{1, \eta}\right) \frac{\partial U_{x}^{\eta}}{\partial \widehat{y}}=-\frac{1}{\mathrm{Kn}_{R}} \sum_{\eta=0}^{n_{F}} J_{2}^{\alpha, \eta} U_{<x y>}^{\eta},
\end{aligned}
$$

where $\mathrm{Kn}_{R}=\frac{c_{0} \tau_{R}^{0}}{2 L}$, and $\alpha$ is the frequency power in the non-equilibrium moment, which is considered to be up to $n_{F}$. The corresponding boundary conditions for this problem are obtained from Eq. (37)

$$
U_{<x y>}^{\alpha}=-\frac{3}{8} \frac{(1-\gamma \beta)}{(\gamma \beta+1)} U_{x}^{\alpha}
$$

Note that by substituting Eq. (47) in the above relation, we can obtain the boundary condition for the energy flux moment.

We derive an expression for $U_{<x y>}^{\beta}$ from Eq. (48)-3 as

$$
U_{<x y>}^{\beta}=-\frac{1}{5} \operatorname{Kn}_{R} \sum_{\gamma=0}^{n_{F}} \sum_{\eta=0}^{n_{F}}\left(J_{2}^{-1}\right)^{\beta, \gamma} F^{\gamma, \eta} \widetilde{U}_{x}^{\eta}
$$

where

$$
F^{\gamma, \eta}=\frac{A_{1}^{\gamma, 1}}{A_{1}^{1,1}} \delta_{\eta}^{1}+\left(A_{1}^{\gamma, \eta}-\frac{A_{1}^{\gamma, 1}}{A_{1}^{1,1}} A_{1}^{1, \eta}\right)
$$

and $\widetilde{U}_{x}^{\eta}$ is the vector of vectorial moments, with the energy flux $Q_{x}$ on its second row, i.e., for $\eta=1$. Moreover, we can obtain the contribution of Fourier-like energy flux from Eq. (48)-2, and define

$$
R_{x}^{\alpha}=-\frac{1}{3} \operatorname{Kn}_{R} \sum_{\eta=0}^{n_{F}}\left(B^{-1}\right)^{\alpha, \eta} \frac{\partial\left(A_{E}^{\eta} U_{0 \mid E}^{\eta}\right)}{\partial U_{0}^{1}} \frac{\partial U_{0}^{1}}{\partial \widehat{x}}
$$

where

$$
B^{\alpha, \eta}=\frac{J_{1}^{\alpha, 1}}{A_{1}^{1,1}} \delta_{\eta}^{1}+\left(J_{1}^{\alpha, \eta}-J_{1}^{\alpha, 1} \frac{A_{1}^{1, \eta}}{A_{1}^{1,1}}\right) .
$$

Note that we have the Fourier law for $R_{x}^{1}$, where we can see the relation between the energy flux and energy gradient.

In order to get a compact form of the governing equations, we subtract the Fourier-like contribution of the fluxes Eq. (50), from the vectorial moments, and define the variable vector

$$
Z_{x}^{\alpha}=\left\{U_{x}^{0}-R_{x}^{0}, Q_{x}-R_{x}^{1}, \ldots, U_{x}^{n_{F}}-R_{x}^{n_{F}}\right\}_{\alpha}
$$

Noting that the energy moment only has a gradient in the $x$-direction, the system of moments in (48) simplifies to an eigenvalue problem as

$$
Z_{x}^{\alpha}=\frac{1}{5} \operatorname{Kn}_{R}^{2} \sum_{\eta=0}^{n_{F}} M^{\alpha, \eta} \frac{\partial^{2} Z_{x}^{\eta}}{\partial \widehat{y}^{2}}
$$

where

$$
M^{\alpha, \eta}=\sum_{\varepsilon, \beta, \gamma}\left(H^{-1}\right)^{\alpha, \varepsilon} A_{2}^{\varepsilon, \beta}\left(J_{2}^{-1}\right)^{\beta, \gamma} F^{\gamma, \eta} .
$$


Solving Eq. (51) gives the energy flux as

$$
Q_{x}=Q_{F}\left(1-\sum_{\gamma} \triangleright^{1, \gamma} C^{\gamma} \cosh \left(\frac{\hat{y}}{\mathrm{Kn}_{R}} \sqrt{\frac{5}{\lambda^{\gamma}}}\right)\right)
$$

where $\triangleright^{\alpha, \gamma}$ is the matrix of eigenvectors, and $\lambda^{\gamma}$ are the eigenvalues of $M^{\alpha, \eta}$, that depends on the considered relaxation time, dispersion relation and the number of frequency powers. Moreover, $C^{\gamma}$ are the constants of integration that need be found from the boundary equation (49), and $Q_{F}$ is the Fourier's heat flux

$$
Q_{F}=-\frac{1}{3} \operatorname{Kn}_{R} \sum_{\eta, \gamma}^{n_{F}} A_{1}^{1, \eta}\left(\left(J_{1}^{-1}\right)^{\eta \gamma} \frac{\partial\left(A_{E}^{\gamma} U_{0 \mid E}^{\gamma}\right)}{\partial U_{0}^{1}}\right) \frac{\partial U_{0}^{1}}{\partial \widehat{x}} .
$$

The system of moments gives a Knudsen layer correction to the Fourier solution. Figure 10 shows the changes in the heat flux due to the increasing number of considered frequency moments $n_{F}$ for two models of relaxation time. In the case of constant relaxation time, the production matrix is diagonal, and increasing the number of frequency powers does not change the solution of the system. In this case, the deviation from the Fourier solution is mainly observed in the vicinity of the crystal boundaries.
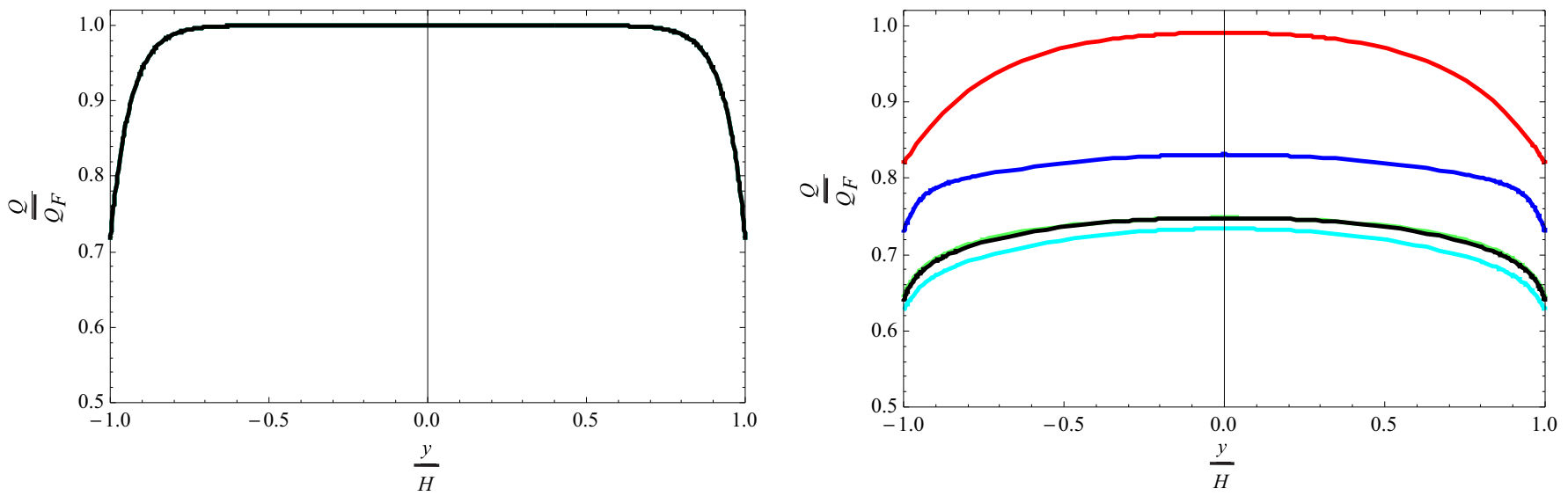

FIG. 10. Heat flux predicted by the system of moments relative to the Fourier's heat flux for the Poiseuille flow of phonons at $\mathrm{Kn}_{R}=\mathrm{Kn}_{N}=0.2, \beta=1$ and $\gamma=0.5$. a) Constant relaxation time model for $n_{F}=2,3,5,6,7$. b) Cross-over relaxation time model for $n_{F}=2,3,5,6,7$.

By considering the frequency dependency in relaxation time we need sufficient number of frequency moments to get a converged solution. By employing 2 frequency powers, i.e., the red line, the rarefaction effects are mainly observed at the boundaries. As we increase $n_{F}$, the deviation from the Fourier's solution is also predicted in the bulk of the phonon gas, and the solution will be more or less the same for $n_{F}=5,6,7$.

The effect of Knudsen number on the energy flux is depicted in Fig. 11-a. As the Knudsen number decreases the energy flux approaches to the plug flow solution, that is predicted by the Fourier law. Increasing rarefaction effects leads to a deviation from the Fourier's solution first at the boundaries, and then in the entire flow field.

Figure 11-b shows the effect of changing the relative portion of specularly reflected and isotropically scattered phonons on the energy flux. The plug flow solution is obtained when the reflection is purely specular $\gamma=1$, which means that boundaries do not have any influence on the bulk. Increasing the portion of isotropically scattered phonons imposes a drag on the gas at the crystal boundary, which leads to Knudsen layer development, and emerging the rarefaction effects in the flow field.

\section{CONCLUSION}

We presented a set of macroscopic equations for phonon transport to describe the thermal properties of a crystal. We employed a quadratic dispersion relation in the finite Brillouin zone to express the dependency of frequency on the wave vector. The collision term in the right hand side of the phonon-Boltzmann equation is described by the Callaway model, where the relaxation time is depending on the frequency of phonons. Using this model, we proposed 

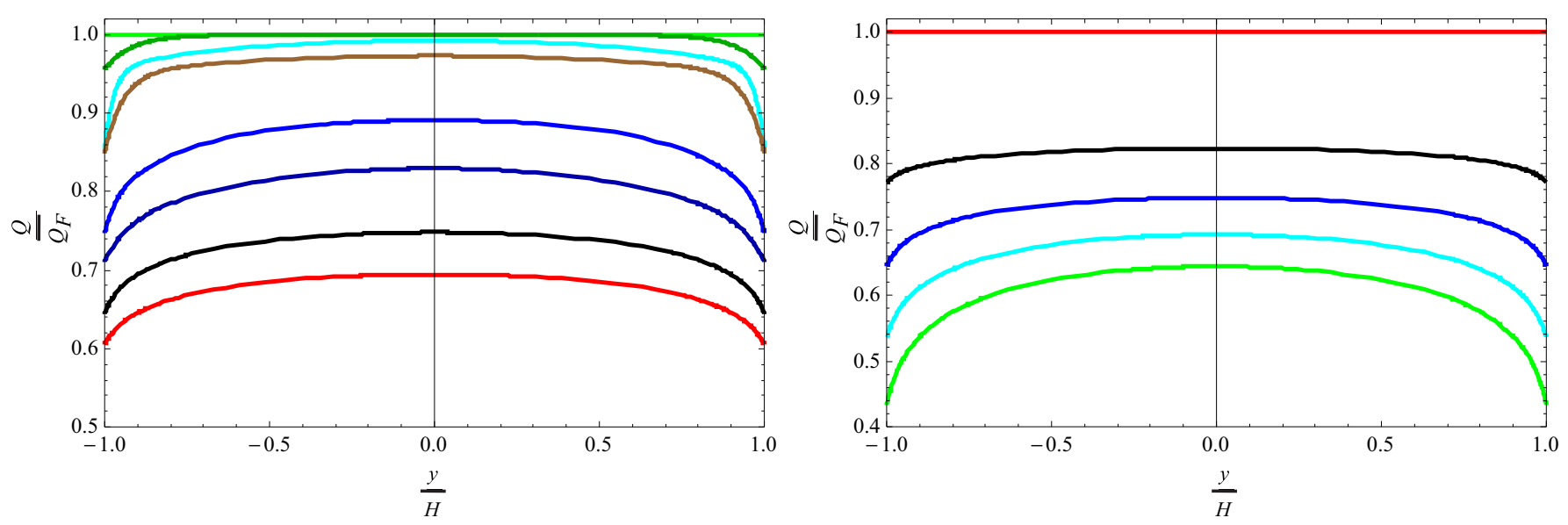

FIG. 11. Heat flux predicted by the system of moments relative to the Fourier's heat flux for the Poiseuille flow of phonons. a) Effect of the Knudsen number $\mathrm{Kn}_{R}=\mathrm{Kn}_{N}=0.0001-0.3, \beta=1$ and $\gamma=0.5$. b) Effect of $\gamma$ on the solution $\gamma=0,0.25,0.5,0.75,1$ and $\mathrm{Kn}_{R}=\mathrm{Kn}_{N}=0.2$

macroscopic moments that depend on the powers of frequency, and the polynomial of phonon's direction vector. Then, the transport equations for macroscopic moments are obtained from the phonon-Boltzmann equation. The closure to this system of equations is provided using the Grad distribution function.

We used the Grad method to provide boundary conditions for the system of moments. We considered three types of microscopic interactions between phonons and crystal boundaries: thermalization, specular reflection and isotropic scattering. The reflection kernel in the proposed boundary model is considered to be the superposition of kernels for these microscopic interaction types.

In order to validate the system of moments, we studied the thermal decay in a one-dimensional body with periodic initial condition. This problem was experimentally studied in Ref. [30]. We observed that the thermal decay deviates from the heat equation already at micron level. We employed different relaxation times in the Callaway model, and observed that by accounting for both low frequency and high frequency phonons in the relaxation time model, we get a good agreement with the reported data in Ref. [30]. As we employed different relaxation time models, we observed that although they all agree in predicting the thermal conductivity (first order rarefaction effect), not all of them can predict the thermal decay curve (higher order rarefaction effect).

As the second application, we solved the one-dimensional Poiseuille flow of phonons in a crystal with adiabatic surface. We observed that in this case, the system of moments predict a Knudsen layer correction to the Fourier's solution.

This study confirms the main role of frequency dependent relaxation time in predicting non-equilbrium heat transport in solids. By comparing our results with the reported data in Ref. [30], we get a validation that the current form of moment equations is capable of capturing the deviation from equilibrium state properly. We now aim to employ the boundary conditions to solve the two-dimensional flow of phonon gas to investigate the effect of thickness on the heat transport in the silicon specimen.

\section{Appendix A: Distribution Function}

Maximizing the entropy density function for phonons under the constraint of our moment definition Eq. (17), leads to

$$
f_{G}=\frac{y}{\exp \left(\frac{h}{k_{B}} \sum_{\beta=0}^{n_{F}} \sum_{m=0}^{n_{D}} \Lambda_{<i_{1} \ldots i_{m}>}^{\beta} \omega^{\beta} n_{<i_{1} \ldots} n_{i_{m}>}\right)-1},
$$

where $\Lambda_{<i_{1} \ldots i_{m}>}^{\beta}$ are the Lagrange multipliers. Comparing this form with the equilibrium distribution function, Eq. (5), suggests that in equilibrium, i.e., $m=0$ and $\beta=1$, all $\Lambda$ are zero, except for

$$
\Lambda^{1}=\frac{1}{T}
$$


Now we separate the equilibrium part from the non-equilibrium contribution, and define

$$
\lambda_{<i_{1} \ldots i_{m}>}^{\beta}=\Lambda_{<i_{1} \ldots i_{m}>}^{\beta}-\frac{1}{T} \delta_{m}^{0} \delta_{\beta}^{1},
$$

that leads to $\lambda^{1}=0$ in the equilibrium. By considering a small deviation from the equilibrium state, we can linearize the distribution function in the non-equilibrium Lagrange multipliers to obtain

$$
f_{G}=f_{E}-\frac{y \exp \left(\frac{\hbar \omega}{k_{B} T}\right)}{\left(\exp \left(\frac{\hbar \omega}{k_{B} T}\right)-1\right)^{2}} \sum_{\beta=0}^{n_{F}} \sum_{m=0}^{n_{D}}\left(\lambda_{<i_{1} \ldots i_{m}>}^{\beta}\left(\frac{\hbar \omega}{k_{B} T}\right)^{\beta} n_{<i_{1} \ldots} n_{i_{m}>}\right) .
$$

The unknown $\lambda_{<i_{1} \ldots i_{m}>}^{\beta}$ are determined as functions of the moments, by substituting this distribution function into Eq. (17), and performing the integration over the Brillouin zone. For this means, first we switch to the spherical coordinates with $d \mathbf{k}=d k d \Omega$, where $\Omega$ as the solid angel, and define the density of states $g(\omega)$ as

$$
g(\omega)=k^{2} \frac{d k}{d \omega}
$$

to only deal with the frequency in our system of equations. Moreover, we introduce non-dimensional functions

$$
X=\frac{\hbar \omega}{k_{B} T} \text { and } \quad G(X)=\frac{c_{0}^{3}}{\omega_{M}^{2}} g(\omega),
$$

where $c_{0}$ is the Debye velocity, and $\omega_{M}$ is the reference frequency

$$
\omega_{M}=\frac{2 c_{0}}{a} \text {. }
$$

By introducing the matrix $I_{\alpha \beta}$ as

$$
I_{\alpha \beta}=\int_{B Z} \frac{X^{\alpha+\beta} \exp (X) G(X)}{(\exp (X)-1)^{2}} d X
$$

and the frequency function

$$
\Phi^{\eta}=\frac{\exp (X)}{(\exp (X)-1)^{2}} \sum_{\beta} X^{\beta} I_{\beta \eta}^{-1},
$$

we obtain the Grad distribution function as in Eq. (22).

\section{Appendix B: Flux term}

By substituting the distribution function in Eq. (23), we get

$$
F_{<i_{1} i_{2} . . i_{n}>}^{\alpha}=c_{0}\left(\frac{\int_{B Z} \frac{\uplus(X) X^{\alpha} G(X)}{\exp (X)-1} d X}{\int_{B Z} \frac{X^{\alpha} G(X)}{\exp (X)-1} d X} u_{0 \mid E}^{\alpha} \delta_{n}^{0}+\right.
$$

$$
\left.\sum_{\substack{\eta=0 \\ \neq E}}^{n_{F}}\left(\int_{B Z} \uplus(X) X^{\alpha} \Phi^{\eta} X_{M}^{\eta-\alpha} G(X) d X\right)\left(u_{<i_{1}, . . i_{n}>}^{\eta}-u_{<i_{1}, . . i_{n}>\mid E}^{\eta}\right)\right)
$$

where the first term only has contribution to the scalar flux term, $n=0$. By separating the above relation for the two cases of scalar, and non-scalar flux terms we get Eq. (24) with

$$
A_{E}^{\alpha}=\frac{\int_{B Z} \frac{\uplus(X) X^{\alpha} G(X)}{\exp (X)-1} d X}{\int_{B Z} \frac{X^{\alpha} G(X)}{\exp (X)-1} d X} \text { and } A_{n_{p}}^{\alpha, \eta}=X_{M}^{\eta-\alpha} \int_{B Z} \uplus(X) X^{\alpha} \Phi^{\eta} G(X) d X .
$$

Note that $\uplus(X)$ is the non-dimensional group velocity

$$
\uplus(X)=\frac{1}{c_{0}} \frac{\partial \omega}{\partial k} .
$$




\section{Appendix C: R-process production term}

By considering a small deviation from the equilibrium state we express the distribution function for $R$-process as

$$
f_{R}=f_{E}-y \frac{X \exp (X)}{(\exp (X)-1)^{2}}\left(\gamma_{R} T-1\right) .
$$

Using the distribution function Eq. (22), and solving Eq. (10) for $\gamma_{R}$, to express this deviation as a function of moments gives

$$
\gamma_{R}=\frac{1}{T}\left(1-\frac{a^{3}}{32 \pi y} \frac{\sum_{\eta=0}^{n_{F}} X_{M}^{\eta+1} \int_{B Z} \frac{X \Phi^{\eta} G(X)}{\Psi_{R}(X)} d X}{\int_{B Z} \frac{X^{2} \exp (X) G(X)}{\Psi_{R}(X)(\exp (X)-1)^{2}} d X} \frac{v_{0}^{\eta}}{\omega_{M}^{\eta}}\right)
$$

Here, $\Psi_{R}$ is the non-dimensional relaxation time for $R$-process

$$
\Psi_{R}(X)=\frac{\tau_{R}\left(\frac{k_{B} T}{\hbar} X\right)}{\tau_{R}^{0}}
$$

Using Eq. (C1) to obtain the $R$-production moment in Eq. (25) we get Eq. (27) with

$$
\begin{aligned}
& J_{0, R}^{\alpha, \eta}=X_{M}^{\eta-\alpha}\left(\int_{B Z} \frac{X^{\alpha} \Phi^{\eta}}{\Psi_{R}(X)} G(X) d X-\frac{\int_{B Z} \frac{X \Phi^{\eta} G(X)}{\Psi_{R}(X)} d X}{\int_{B Z} \frac{X^{2} \exp (X) G(X)}{\Psi_{R}(X)(\exp (X)-1)^{2}} d X} \int_{B Z} \frac{X^{\alpha+1} \exp (X) G(X)}{\Psi_{R}(X)(\exp (X)-1)^{2}} d X\right), \\
& J_{n, R}^{\alpha, \eta}=X_{M}^{\eta-\alpha} \int_{B Z} \frac{X^{\alpha} \Phi^{\eta}}{\Psi_{R}(X)} G(X) d X .
\end{aligned}
$$

\section{Appendix D: N-Process production term}

Similar to the $R$-process, first we assume a small deviation from the equilibrium state to express $f_{N}$ as

$$
f_{N}=f_{E}-y\left(\frac{X \exp (X)}{(\exp (X)-1)^{2}}\left(\gamma_{N} T-1\right)+\frac{\exp (X) \sqrt{\uplus(X) G(X)}}{(\exp (X)-1)^{2}} \frac{\hbar \omega_{M}}{k_{B} c_{0}} \xi_{j} n_{j}\right) .
$$

Then we solve Eq. (11) for $\gamma_{N}$ and $\xi_{i}$ to get

$$
\gamma_{N}=\frac{1}{T}\left(1-\frac{a^{3}}{32 \pi y} \frac{\sum_{\eta=0,}^{n_{F}} X_{M}^{\eta+1} \int_{B Z} \frac{X \Phi^{\eta} G(X)}{\Psi_{N}(X)} d X}{\int_{B Z} \frac{X^{2} \exp (X) G(X)}{\Psi_{N}(X)(\exp (X)-1)^{2}} d X} \frac{v_{0}^{\eta}}{\omega_{M}^{\eta}}\right)
$$

and

$$
\xi_{i}=-\frac{3 a^{4}}{64 \pi} \frac{k_{B}}{\hbar y} \sum_{\eta} \frac{\int \frac{\sqrt{\uplus(X)} \Phi^{\eta} X_{M}^{\eta+1} G(X)^{\frac{3}{2}}}{\Psi_{N}(X)} d X}{\int \frac{\uplus(X) \exp (X) G(X)^{2} d X}{\Psi_{N}(X)(\exp (X)-1)^{2}}} \frac{u_{i}^{\eta}}{\omega_{M}^{\eta}} .
$$

Here, $\Psi_{N}$ is the non-dimensional relaxation time for $N$-process

$$
\Psi_{N}(X)=\frac{\tau_{N}\left(\frac{k_{B} T}{\hbar} X\right)}{\tau_{N}^{0}}
$$


By accessing to the distribution function for the $N$-process, we can obtain the production moments in Eq. (28) with

$$
\begin{aligned}
& J_{0, N}^{\alpha, \eta}=X_{M}^{\eta-\alpha}\left(\int_{B Z} \frac{X^{\alpha} \Phi^{\eta}}{\Psi_{N}(X)} G(X) d X-\frac{\int_{B Z} \frac{X \Phi^{\eta} G(X)}{\Psi_{N}(X)} d X}{\int_{B Z} \frac{X^{2} \exp (X) G(X)}{\Psi_{N}(X)(\exp (X)-1)^{2}} d X} \int_{B Z} \frac{X^{\alpha+1} \exp (X) G(X)}{(\exp (X)-1)^{2} \Psi_{N}(X)} d X\right) \\
& J_{1, N}^{\alpha, \eta}=X_{M}^{\eta-\alpha}\left(\int_{B Z} \frac{X^{\alpha} \Phi^{\eta}}{\Psi_{N}(X)} G(X) d X-\frac{\int_{B Z} \frac{\sqrt{\uplus(X) \Phi^{\eta} G(X)^{\frac{3}{2}}}}{\Psi_{N}(X)} d X}{\int_{B Z} \frac{\exp (X) \uplus(X) G(X)^{2}}{\Psi_{N}(X)(\exp (X)-1)^{2}} d X} \int_{B Z} \frac{X^{\alpha} \sqrt{\uplus(X)} \exp (X) G(X)^{\frac{3}{2}}}{(\exp (X)-1)^{2} \Psi_{N}(X)} d X\right) \\
& J_{n, N}^{\alpha, \eta}=X_{M}^{\eta-\alpha} \int_{B Z} \frac{X^{\alpha} \Phi^{\eta}}{\Psi_{N}(X)} G(X) d X .
\end{aligned}
$$

\section{Appendix E: Distribution function for the isotropic scattering}

As mentioned in IV A, for this type of interaction the impacting phonon with the surface is reflected in a random direction

$$
f_{S c}=\frac{1}{\pi c} \int_{n_{k} \nu_{k}<0} c\left(-n_{k} \nu_{k}\right) f_{G} d \Omega
$$

Using Grad distribution function in the above relation, and further simplification gives

$$
f_{S c}=f_{E}-\frac{a^{3}}{32 \pi} \sum_{\eta, m} \frac{(2 m+1) ! !}{m !} \Phi^{\eta} X_{M}^{\eta+1}\left(\frac{u_{<j_{1}, . . j_{m}>}^{\eta}-u_{<j_{1}, . . j_{m}>\mid E}^{\eta}}{\omega_{M}^{\eta}}\right) \int_{k_{i} \nu_{i}<0} n_{j_{1} \ldots} n_{j_{m}} n_{k} \nu_{k} d \Omega
$$

Note that here we use the direction vector with trace to simplify the integration over the half space. Without losing the generality, we consider a surface with the normal in the $z$-direction, so that the normal unit vector to the surface reads $\nu_{i}=\{0,0,1\}_{i}$. Moreover, we use the normal-tangential framework with respect to the surface, where the direction vector can be written as $n_{i}=\left\{\nu, \tau_{A}\right\}_{i}$ with $\nu=n_{i} \nu_{i}=\cos \theta$ and $\tau_{A}=\{\sin \theta \cos \phi, \sin \theta \sin \phi\}$. Using this notation we can express the tensorial moments with normal and tangential components. For example the second order tensor $u_{<i j>}$ can be written as

$$
u_{<i j>}^{\alpha}=\left[\begin{array}{ll}
u_{<A B>}^{\alpha} & u_{<A \nu>}^{\alpha} \\
u_{<B \nu>}^{\alpha} & u_{<\nu \nu>}^{\alpha}
\end{array}\right]_{i j}
$$

where the capital indices, $A$ and $B$ denote the tangential directions. Note that the trace-free condition reads $u_{<A A>}^{\alpha}+$ $u_{<\nu \nu>}^{\alpha}=0$. Following the same method, the higher order tensors can be expressed as

$$
\left(\frac{u_{<j_{1}, . . j_{m}>}^{\eta}-u_{<j_{1}, . . j_{m}>\mid E}^{\eta}}{\omega_{M}^{\eta}}\right) n_{j_{1} \ldots} n_{j_{m}}=\sum_{r=0}^{m} \frac{m !}{r !(m-r) !}
$$$$
\left(\frac{u_{<A_{1} \ldots A_{r} \nu \ldots \nu>}^{\eta}-u_{<A_{1} \ldots A_{r} \nu \ldots \nu>\mid E}^{\eta}}{\omega_{M}^{\eta}}\right) \tau_{A_{1} \ldots \tau_{A_{r}} \nu^{m-r}}
$$

Using this relation in Eq. (E1) we can get

$$
\begin{aligned}
f_{S c}=f_{E}-\frac{a^{3}}{32 \pi^{2}} \sum_{\eta, m} \frac{(2 m+1) ! !}{m !} \Phi^{\eta} X_{M}^{\eta+1} \sum_{r=0}^{m} \frac{m !}{r !(m-r) !} & \left(\frac{u_{<A_{1} \ldots A_{r} \nu \ldots \nu>}^{\eta}-u_{<A_{1} \ldots A_{r} \nu \ldots \nu>\mid E}^{\eta}}{\omega_{M}^{\eta}} \int_{\nu<0} \tau_{A_{1} \ldots \tau_{A_{r}} \nu}^{m-r+1} d \Omega .\right.
\end{aligned}
$$

The integral only has non-zero value for even numbers of $r$, which can be written as

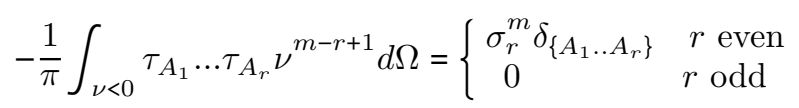


here $\delta_{\left\{A_{1} . . A_{r}\right\}}$ is the generalized tangential unit tensor, that has $(r-1)$ !! terms. For example

$$
\delta_{\{A B\}}=\delta_{A B} \quad \text { and } \quad \delta_{\{A B C D\}}=\delta_{A B} \delta_{C D}+\delta_{A C} \delta_{B D}+\delta_{A D} \delta_{B C} .
$$

By taking the traces from Eq. (E2), we obtain

$$
\sigma_{r}^{m}=(-1)^{m-r} \frac{\Gamma\left(1+\frac{m-r}{2}\right) \Gamma\left(1+\frac{r}{2}\right)}{r ! ! \Gamma\left(2+\frac{m}{2}\right)},
$$

where $\Gamma$ is the Gamma function. Using the tensorial calculation, we simplify the product of the tonsorial moments with the unit tangential tensors as

$$
\left(\frac{u_{<A_{1} \ldots A_{r} \nu \ldots \nu>}^{\eta}-u_{<A_{1} \ldots A_{r} \nu \ldots \nu>\mid E}^{\eta}}{\omega_{M}^{\eta}}\right) \delta_{\left\{A_{1} \ldots A_{r}\right\}}=(r-1) ! !(-1)^{\frac{r}{2}}\left(\frac{u_{<v \ldots \nu>}^{\eta}-u_{<v \ldots \nu>\mid E}^{\eta}}{\omega_{M}^{\eta}}\right)_{\mid m} .
$$

Here, the subscript $m$ denotes the moment with $m$ indices. The distribution function for the isotropic scattering can now be written as

$$
f_{S c}=f_{E}+\frac{a^{3}}{32 \pi} \sum_{\eta, m} \Phi^{\eta} X_{M}^{\eta+1} \mu^{m}\left(\frac{u_{<v \ldots \nu>}^{\eta}-u_{<v \ldots \nu>\mid E}^{\eta}}{\omega_{M}^{\eta}}\right)_{\mid m},
$$

where

$$
\mu^{m}=\sum_{\substack{r=0 \\ r \text { even }}}^{m}(-1)^{m-\frac{r}{2}} \frac{(r-1) ! !}{r ! !} \frac{(2 m+1) ! !\left(\frac{r}{2}\right) !}{r !(m-r) !} \frac{\Gamma\left(1+\frac{m-r}{2}\right)}{\Gamma\left(2+\frac{m}{2}\right)}
$$

Equation (E4) relates the distribution function for the particles that isotropically scattered from the surface to the normal components of the non-equilibrium moments.

\section{Appendix F: Obtaining Eq. (34)}

Starting from Eq. (E2), first we need to substitute the Grad distribution function. In here, the directional integration has a different sign compared to Eq. (E2),

$$
-\frac{1}{\pi} \int_{\nu>0} \tau_{A_{1}} \ldots \tau_{A_{r}} \nu^{m-r+1} d \Omega=\left\{\begin{array}{cl}
\xi_{r}^{m} \delta_{\left\{A_{1} . . A_{r}\right\}} & r \text { even } \\
0 & r \text { odd }
\end{array}\right.
$$

where $\xi_{r}^{m}=\left|\sigma_{r}^{m}\right|$. The right hand side Eq. (33) can be written as

$$
\begin{aligned}
\int_{\nu>0} \omega^{\alpha} \tau_{B_{1}} \ldots \tau_{B_{s}} \nu^{m-s+1} f_{G} d \mathbf{k}= & \frac{8 \pi \omega_{M}^{\alpha}}{a^{3} X_{M}^{\alpha+1}}\left(\int_{B Z} X^{\alpha} f_{E} G(X) d X\right) \xi_{s}^{m} \delta_{\left\{B_{1} \ldots B_{s}\right\}} \\
& +\frac{1}{4} \sum_{n} \sum_{r=0}^{n} \frac{(2 n+1) ! !}{r !(n-r) !} \xi_{r+s}^{n+m}\left(u_{<A_{1} \ldots A_{r} \nu_{1} \ldots \nu_{n-r}>}^{\alpha}-u_{\left.<A_{1} \ldots A_{r} \nu_{1} \ldots \nu_{n-r}>\mid E\right)}^{\alpha} \delta_{\left\{A_{1} \ldots A_{r} B_{1} \ldots B_{s}\right\}} .\right.
\end{aligned}
$$

The contribution for the diffusive particles on the left hand side of Eq. (33) can be written as

$$
(1-\beta) \int_{\nu>0} \omega^{\alpha} \tau_{B_{1}} \ldots \tau_{B_{s}} \nu^{m-s+1} f_{E}\left(T_{s}\right) d \mathbf{k}=\frac{8 \pi \omega_{M}^{\alpha}}{a^{3} X_{M}^{\alpha+1}}(1-\beta)\left(\int_{B Z} X^{\alpha} f_{E}\left(T_{s}\right) G(X) d X\right) \xi_{s}^{m} \delta_{\left\{B_{1} \ldots B_{s}\right\}} .
$$

For the specularly reflected particles we have

$$
\begin{aligned}
\beta \gamma \int_{\nu>0} \omega^{\alpha} \tau_{B_{1}} \ldots \tau_{B_{s}} \nu^{m-s+1} f_{S} d \mathbf{k} & =\beta \gamma\left(\frac{8 \pi \omega_{M}^{\alpha}}{a^{3} X_{M}^{\alpha+1}}\left(\int_{B Z} X^{\alpha} f_{E} G(X) d X\right) \sigma_{s}^{m} \delta_{\left\{B_{1} \ldots B_{s}\right\}}\right. \\
+ & \left.\frac{1}{4} \sum_{n} \sum_{r=0}^{n} \frac{(2 n+1) ! !}{r !(n-r) !} \sigma_{r+s}^{n+m}\left(u_{<A_{1} \ldots A_{r} \nu_{1} \ldots \nu_{n-r}>}^{\alpha}-u_{<A_{1} \ldots A_{r} \nu_{1} \ldots \nu_{n-r}>\mid E}^{\alpha}\right) \delta_{\left\{A_{1} \ldots A_{r} B_{1} \ldots B_{s}\right\}}\right)
\end{aligned}
$$


and for the isotropic scattering, by using Eq. (E4) we get

$$
\begin{aligned}
\beta(1-\gamma) \int_{\nu>0} \omega^{\alpha} \tau_{B_{1}} \ldots \tau_{B_{s}} \nu^{m-s+1} f_{S c} d \mathbf{k}=\beta(1-\gamma)\left(\frac{8 \pi \omega_{M}^{\alpha}}{a^{3} X_{M}^{\alpha+1}}\right. & \left.\left(\int_{B Z} X^{\alpha} f_{E} G(X) d X\right)+\frac{1}{4} \sum_{n} \mu^{\alpha}\left(u_{<\nu_{1} \ldots \nu_{n}>}^{\alpha}-u_{<\nu_{1} \ldots \nu_{n}>\mid E}^{\alpha}\right)\right) \xi_{s}^{m} \delta_{\left\{B_{1} \ldots B_{s}\right\}} .
\end{aligned}
$$

Substituting these relations in Eq. (33), we obtain Eq. (34).

\section{ACKNOWLEDGMENTS}

This research was supported by the Natural Sciences and Engineering Council (NSERC).

[1] G. Karniadakis, A. Beskok, N. Aluru, Microflows and Nanoflows: Fundamentals and simulation, Springer, New York, 2005.

[2] G. A. Bird, Molecular gas dynamics and the direct simulation of gas flows, Oxford university press, Oxford, 1994.

[3] V. Romano, A. Rusakov, 2d numerical simulations of an electron-phonon hydrodynamical model based on the maximum entropy principle, Comput. Methods Appl. Mech. Eng. 199 (2010) 2741-2751.

[4] C. Kittel, Introduction to solid state physics, John Wiley \& sons, Hoboken, 1996.

[5] D. Snoke, Solid state physics essential concepts, Addison-Wesley, San Francisco, 2009.

[6] W. Dreyer, H. Struchtrup, Heat pulse experiments revisited, Contin. Mech. Thermodyn. 5 (1993) 3-50.

[7] R. J. Gutfeld, A. H. Nethercot, Heat pulses in quartz and sapphire at low temperatures, Phys. Rev. Lett. 12 (1964) 641.

[8] R. A. Guyer, J. A. Krumhansl, Thermal conductivity, second sound and phonon hydrodynamic phenomena in non-metallic crystals, Phys. Rev. 148 (1966) 778.

[9] R. Peierls, Zur kinetischen theorie der wärmeleitung in kristallen, Annalen der physik (1929) 1055-1101.

[10] L. Chaput, Direct solution to the linearized phonon boltzmann equation, Phys. Rev. Lett. 110 (2013) 265506.

[11] A. J. H. McGaughey, M. Kaviany, Thermal conductivity decomposition and analysis using molecular dynamics simulations. part i. lennard-jones argon, Int. J. Heat Mass Transfer 27 (2004) 1783-1798.

[12] A. Mohammadzadeh, E. Roohi, H. Niazmand, A parallel dsmc investigation of monatomic/diatomic gas flows in a micro/nano cavity, Num. Heat Transfer 63 (2013) 305-325.

[13] J.-P. M. Peraud, N. Hadjiconstantinou, Efficient simulation of multidimensional phonon transport using energy-based variance-reduced monte carlo formulations, Phys. Rev. B 84 (2011) 205331.

[14] J.-P. M. Peraud, N. Hadjiconstantinou, An alternative approach to efficient simulation of micro/nanoscale phonon transport, Appl. Phys. Lett. 101 (2012) 153114.

[15] J. Callaway, Model for lattice thermal conductivity at low temperatures, Phys. Rev. 113 (1959) $1046-1051$.

[16] S. Chapman, T. Cowling, The mathematical theory of nonuniform sases, Cambridge University Press, 1970.

[17] M. I. Flik, B. I. Choi, K. E. Goodson, Heat transfer regimes in microstructures, ASME J. Heat Transfer 114 (1992) 666-674.

[18] A. Majumdar, Microscale energy transport in solids, Taylor and Francis, Washington DC, 1998.

[19] G. Chen, Particularities of heat conduction in nanostructures, J. Nanopart. Res. 2 (2000) 199-204.

[20] D. Joseph, L. Preziosi, Heat waves, Rev. Mod. Phys. 61.

[21] R. A. Guyer, J. A. Krumhansl, Dispersion relation for second sound in solids, Phys. Rev. 133 (1964) 14117.

[22] R. A. Guyer, J. A. Krumhansl, Solution of the linearized phonon boltzmann equation, Phys. Rev. 148 (1966) 7668.

[23] H. Grad, On the kinetic theory of rarefied gases, Commun. Pure Appl. Math. 2 (1949) 331-407.

[24] W. Dreyer, Maximisation of the entropy in non-equilibrium, J. Phys. A: Math. Gen. 20 (1987) 6505.

[25] H. Struchtrup, Macroscopic transport equations for rarefied gas flows, Springer, New York, 2005.

[26] A. Rana, A. Mohammadzadeh, H. Struchtrup, A numerical study of the heat transfer through a rarefied gas confined in a microcavity, Contin. Mech. Thermodyn. 27 (2015) 433-446.

[27] A. Mohammadzadeh, A. Rana, H. Struchtrup, Thermal stress vs. thermal transpiration: A competition in thermally driven cavity flows, Phys. Fluids 27 (2015) 112001.

[28] A. Rana, M. Torrilhon, H. Struchtrup, A robust numerical method for the r13 equations of rarefied gas dynamics: Application to lid driven cavity, J. Compu. Phys. 236 (2013) 169-186.

[29] M. Fryer, H. Struchtrup, Moment model and boundary conditions for energy transport in the phonon gas, Continuum Mech. Thermodyn. 26 (2013) 593-618.

[30] J. A. Johnson, A. A. Maznev, J. Cuffe, J. K. Eliason, A. J. Minnich, T. Kehoe, C. M. S.Torres, G. Chen, K. A. Nelson, Direct measurement of room-temperature nondiffusive thermal transport over micron distances in a silicon membrane, Phys. Rev. Lett. 110 (2013) 025901.

[31] R. Debye, Zur theorie tier spezifischen wgrmen, Ann. Phys. 39. 
[32] A. Einstein, Die plancksche theorie der strahlung und die theorie der spezifischen warme, Ann. d. Phys. 22.

[33] E. Pop, R. W. Dutton, K. E. Goodson, Analytic band monte carlo model for electron transport in si including acoustic and optical phonon dispersion, J. Appl. Phys. 96 (2004) 4998.

[34] P. Bhatnagar, E. Gross, M. Krook, A model for collision processes in gases. i. small amplitude processes in charged and neutral one-component systems, Phys. Rev. 94 (1954) 511-525.

[35] N. Mingo, L. Yang, D. Li, A. Majumdar, Predicting the thermal conductivity of si and ge nanowires, Nano Letters 3 (2003) $1713-1716$

[36] A. Ward, D. A. Broido, Intrinsic phonon relaxation times from first-principles studies of the thermal conductivities of si and ge, Phys. Rev. B 81 (2010) 085205.

[37] P. G. Klemens, Solid state physics, Academic press, New York, 1958.

[38] M. G. Holland, Analysis of lattice thermal conductivity, Phys. Rev. 132 (1963) 2461.

[39] J. Maxwell, On stresses in rarified gases arising from inequalities of temperature, Philos. Trans. R. Soc. Lond. 170 (1879) $231-256$.

[40] H. Struchtrup, Maxwell boundary condition and velocity dependent accommodation coefficient, Phys. Fluids. 25 (2013) 112001.

[41] A. Mohammadzadeh, A. Rana, H. Struchtrup, Dsmc and r13 modeling of the adiabatic surface, Accepted in Int. J. Thermal Sciences.

[42] M. Torrilhon, H. Struchtrup, Boundary conditions for regularized 13-moment-equations for micro-channel-flows, Accepted in Int. J. Thermal Sciences 227 (2011) 1982.

[43] A. A. Maznev, J. A. Johnson, K. A. Nelson, Onset of nondiffusive phonon transport in transient thermal grating decay, Phys. Rev. B 84 (2011) 195206. 